ALEA, Lat. Am. J. Probab. Math. Stat. 18, 81-106 (2021)

DOI: 10.30757/ALEA.v18-05

\title{
Cross-multiplicative coalescent processes and applications
}

\author{
Yevgeniy Kovchegov, Peter T. Otto and Anatoly Yambartsev \\ Department of Mathematics \\ Oregon State University \\ Corvallis, OR 97331, USA \\ E-mail address: kovchegy@math.oregonstate.edu \\ URL: https://math.oregonstate.edu/people/view/kovchegy \\ Department of Mathematics \\ Willamette University \\ Salem, OR 97302, USA \\ E-mail address: potto@willamette.edu \\ URL: https://willamette.edu/ potto/ \\ Department of Statistics \\ Institute of Mathematics and Statistics \\ University of São Paulo \\ Rua do Matão 1010, CEP 05508-090, \\ São Paulo SP, Brazil \\ E-mail address: yambar@ime.usp.br \\ URL: https://www.ime.usp.br/ yambar/
}

\begin{abstract}
We introduce and analyze a novel type of coalescent processes called cross-multiplicative coalescent that models a system with two types of particles, $A$ and $B$. The bonds are formed only between the pairs of particles of opposite types with the same rate for each bond, producing connected components made of particles of both types. We analyze and solve the Smoluchowski coagulation system of equations obtained as a hydrodynamic limit of the corresponding MarcusLushnikov process. We establish that the cross-multiplicative kernel is a gelling kernel, and find the gelation time. As an application, we derive the limiting mean length of a minimal spanning tree on a complete asymmetric bipartite graph with independent edge weights, distributed uniformly over $[0,1]$.
\end{abstract}

Received by the editors June 24th, 2020; accepted October 3rd, 2020.

2010 Mathematics Subject Classification. 60K35, 82B27, 60C05, 05C80.

Key words and phrases. coalescent processes, gelation, Smoluchowski coagulation equations, Marcus-Lushnikov processes, minimal spanning tree, Erdős-Rényi random graph model, density dependent population processes.

This work was supported by the FAPESP award 2016/19286-0 and by the NSF award DMS1412557. 


\section{Introduction}

The coalescence dynamics of clusters with multidimensional weight (mass) vectors was originally considered in Krapivsky and Ben-Naim (1996) and Vigil and Ziff (1998) in the context of aggregation kinetics with applications to aerosol dynamics and copolymerization kinetics. In this paper, we consider a coalescent process whose clusters have vector-valued weights in $\mathbb{R}_{+}^{2}$. The coalescent process begins with $\alpha[n]=\alpha n+o(\sqrt{n})$ singletons of weight $\left[\begin{array}{l}1 \\ 0\end{array}\right]$ and $\beta[n]=\beta n+o(\sqrt{n})$ singletons of weight $\left[\begin{array}{l}0 \\ 1\end{array}\right]$. This continuous time Markov process evolves as follows. Each pair of clusters with respective weight vectors $\mathbf{i}=\left[\begin{array}{l}i_{1} \\ i_{2}\end{array}\right]$ and $\mathbf{j}=\left[\begin{array}{l}j_{1} \\ j_{2}\end{array}\right]$ has the rate $K(\mathbf{i}, \mathbf{j}) / n$ for coalescing into a cluster of weight $\mathbf{i}+\mathbf{j}$, where

$$
K(\mathbf{i}, \mathbf{j})=i_{1} j_{2}+i_{2} j_{1}
$$

is the cross-multiplicative coalescent kernel governing the coalescent process. Such process will be called the cross-multiplicative coalescent process.

As a physical model, one may consider a system with two types of particles, $A$ and $B$. The process begins with $\alpha[n]$ particles of type $A$ and $\beta[n]$ particles of type $B$. Each particle interacts only with the particles of opposite type, with which it may form a bond. The bonds are formed independently, each with rate $1 / n$. Thus, the bonds may be formed only between the pairs of particles of opposite types, producing connected components (clusters). In these clusters, each pair of neighbor vertices will be of opposite type. The model can be interpreted as a bond percolation model on a complete bipartite graph $K_{\alpha[n], \beta[n]}$ with the probability of an edge being open $p=1-e^{-t / n}$ increasing from zero to one as the time $t$ increases from zero to infinity. See Subsection 4.1.

Let $\zeta_{i_{1}, i_{2}}^{[n]}(t)$ denote the number of the components of weight $\left[\begin{array}{l}i_{1} \\ i_{2}\end{array}\right]$ at time $t$. The hydrodynamic limit

$$
\lim _{n \rightarrow \infty} \sup _{s \in[0, T]}\left|n^{-1} \zeta_{i_{1}, i_{2}}^{[n]}(s)-\zeta_{i_{1}, i_{2}}(s)\right|=0 \quad \text { a.s. }
$$

for an arbitrary $T>0$ is established in Subsection 5.2 via the weak convergence results in Kurtz (1981) and Ethier and Kurtz (1986) for density dependent population processes. The limiting functions $\zeta_{i_{1}, i_{2}}(t)$ indexed by $\mathbb{Z}_{+}^{2} \backslash\{(0,0)\}$ are expressed as the solutions of the following modified Smoluchowski coagulation system of differential equations

$$
\frac{d}{d t} \zeta_{i_{1}, i_{2}}(t)=-\left(\beta i_{1}+\alpha i_{2}\right) \zeta_{i_{1}, i_{2}}(t)+\frac{1}{2} \sum_{\substack{\ell_{1}, k_{1}: \ell_{1}+k_{1}=i_{1}, \ell_{2}, k_{2}: \ell_{2}+k_{2}=i_{2}}}\left(\ell_{1} k_{2}+\ell_{2} k_{1}\right) \zeta_{\ell_{1}, \ell_{2}}(t) \zeta_{k_{1}, k_{2}}(t)
$$

with the initial conditions $\zeta_{i_{1}, i_{2}}(0)=\alpha \delta_{1, i_{1}} \delta_{0, i_{2}}+\beta \delta_{0, i_{1}} \delta_{1, i_{2}}$. The above coagulation system has a unique solution as established in Theorem 3.2 of Section 3. This solution enables us to prove gelation occurs in the cross-multiplicative coalescent process and find the gelation time. See Section 3, Corollary 3.7. As a particular application we find the asymptotic limit for the mean length of a minimal spanning tree for the complete bipartite graph $K_{\alpha[n], \beta[n]}$ with partitions of sizes $\alpha[n]=$ $\alpha n+o(\sqrt{n})$ and $\beta[n]=\beta n+o(\sqrt{n})$. See Section 4 . 
The paper is organized as follows. Section 2 provides the background on coalescent processes and gelation. In Section 3 the cross-multiplicative coalescent process and the corresponding Marcus-Lushnikov process are analyzed. Section 4 gives an application of the cross-multiplicative coalescent process in minimal spanning trees. Finally, in Section 5, the weak convergence results from Kurtz (1981) and Ethier and Kurtz (1986) are applied to Marcus-Lushnikov processes with the cross-multiplicative kernel. The paper concludes with a discussion in Section 6 .

\section{Background on coalescent processes and gelation}

A general finite coalescent process begins with $n$ singletons (clusters of mass one). The cluster formation is governed by a symmetric collision rate kernel $K(i, j)=$ $K(j, i)>0$. Specifically, a pair of clusters with masses (weights) $i$ and $j$ coalesces at the rate $K(i, j) / n$, independently of the other pairs, to form a new cluster of mass $i+j$. The process continues until there is a single cluster of mass $n$. See Pitman (2006); Aldous (1999); Bertoin (2006); Berestycki (2009); Evans and Pitman (1998) and references therein.

Formally, for a given $n$ consider the space $\mathcal{P}_{[n]}$ of partitions of $[n]=\{1,2, \ldots, n\}$. Let $\Pi_{0}^{(n)}$ be the initial partition in singletons, and $\Pi_{t}^{(n)}(t \geq 0)$ be a strong Markov process such that $\Pi_{t}^{(n)}$ transitions from partition $\pi \in \mathcal{P}_{[n]}$ to $\pi^{\prime} \in \mathcal{P}_{[n]}$ with rate $K(i, j) / n$ provided that partition $\pi^{\prime}$ is obtained from partition $\pi$ by merging two clusters of $\pi$ of weights $i$ and $j$. If $K(i, j) \equiv 1$ for all positive integer masses $i$ and $j$, the process $\Pi_{t}^{(n)}$ is known as Kingman's $n$-coalescent process. If $K(i, j)=i+j$ the process is called $n$-particle additive coalescent. Finally, if $K(i, j)=i j$ the process is called $n$-particle multiplicative coalescent. The so called Marcus-Lushnikov process

$$
\mathbf{M L}_{n}(t)=\left(\zeta_{1}^{[n]}(t), \zeta_{2}^{[n]}(t), \ldots, \zeta_{n}^{[n]}(t), 0,0, \ldots\right)
$$

is an auxiliary process to the corresponding coalescent process that keeps track of the numbers of clusters in each weight category. Here we let $\zeta_{k}^{[n]}(t)$ denote the number of clusters of mass $k$ in a coalescent process of $n$ particles at time $t \geq 0$. See Marcus (1968) and Lushnikov (1978) for the original papers. The latter work considered the gelation phenomenon emerging in some of the MarcusLushnikov processes. The Marcus-Lushnikov process does not differentiate between the clusters of the same weight, and therefore does not keep track of the merger history of the $n$-particle coalescent process.

The deterministic dynamics of the limiting fractions $\zeta_{k}(t)=\lim _{n \rightarrow \infty} \frac{\zeta_{k}^{[n]}(t)}{n}$ of clusters of size $k$ is described by the Smoluchowski system of coagulation equations Smoluchowski (1916) or by its modified version, the Flory equations, named after Flory (1953). See Jeon (1998); Norris (1999); Fournier and Giet (2004); Fournier and Laurençot (2009). The general system of Smoluchowski coagulation equations with a positive symmetric kernel $K(i, j)$ is the following mean-field approximation of coalescent dynamics

$$
\frac{d}{d t} \zeta_{j}=-\zeta_{j} \sum_{i=1}^{\infty} K(i, j) \zeta_{i}+\frac{1}{2} \sum_{i=1}^{j-1} K(i, j-i) \zeta_{i} \zeta_{j-i} \quad(k=1,2, \ldots)
$$


One of the important questions in the theory of Smoluchowski equations is whether the conservation of mass property

$$
\sum_{j=1}^{\infty} j \zeta_{j}(t)=\sum_{j=1}^{\infty} j \zeta_{j}(0)
$$

holds for all $t \geq 0$, or if there exists a time $T_{\text {gel }}<\infty$ after which the total mass $\sum_{j=1}^{\infty} j \zeta_{j}$ begins to dissipate.

2.1. Gelation. The phenomenon of loosing total mass after a certain finite time $T_{\text {gel }}$ is called gelation. Time $T_{\text {gel }}>0$, if finite, is called the gelation time. The kernel function $K(\cdot, \cdot)$ for which such $T_{g e l}<\infty$ is called the gelling kernel. Informally, the gelation time corresponds to formation of a giant cluster called the gel. The gelation phenomenon was studied extensively in the coagulations equations literature. See Aldous (1998, 1999); van Dongen and Ernst (1986); Jeon (1999); Lushnikov (1978) and references therein. Here, we would like to summarize some (but not all) of the concepts and results concerning the gelation phenomenon.

Consider a general system (2.2) of Smoluchowski coagulation equations with a positive symmetric kernel $K(i, j)$, and given initial conditions $\zeta_{j}(0)$. Then, following McLeod (1962), we use the Smoluchowski equations (2.2) to obtain

$$
\begin{aligned}
\frac{d}{d t} \sum_{j=1}^{\infty} j \zeta_{j}=\sum_{j=1}^{\infty} j \frac{d}{d t} \zeta_{j} & =-\sum_{i, j=1}^{\infty} j K(i, j) \zeta_{j} \zeta_{i}+\frac{1}{2} \sum_{j=1}^{\infty} \sum_{i=1}^{j-1}(i+(j-i)) K(i, j-i) \zeta_{i} \zeta_{j-i} \\
& =-\sum_{i, j=1}^{\infty} j K(i, j) \zeta_{j} \zeta_{i}+\frac{1}{2} \sum_{i, j=1}^{\infty}(i+j) K(i, j) \zeta_{i} \zeta_{j}=0
\end{aligned}
$$

provided convergence of $\sum_{i, j=1}^{\infty} j K(i, j) \zeta_{j} \zeta_{i}$. Thus, letting the gelation time be defined via the following critical transition,

$$
T_{g e l}:=\inf \left\{t>0: \sum_{i, j=1}^{\infty} j K(i, j) \zeta_{j}(t) \zeta_{i}(t)=\infty\right\},
$$

we have $\frac{d}{d t} \sum_{j=1}^{\infty} j \zeta_{j}=0$ for $t \in\left[0, T_{\text {gel }}\right)$, which in turn implies (2.3) for $t \in\left[0, T_{\text {gel }}\right)$.

Suppose the hydrodynamic limit $\lim _{n \rightarrow \infty} \frac{\zeta_{k}^{[n]}(t)}{n}=\zeta_{k}(t)$ is established for the Marcus-Lushnikov process with the given kernel $K(i, j)$, where $\zeta_{k}(t)$ is the solution of a coagulation system of equations. See Norris (1999); Fournier and Giet (2004); Fournier and Laurençot (2009). Then the definition of gelation time in formula (2.4) is replaced with

$$
T_{g e l}:=\inf \left\{t>0: \sum_{j=1}^{\infty} j \zeta_{j}(t)<\sum_{j=1}^{\infty} j \zeta_{j}(0)\right\} .
$$

While (2.4) relies on the explosion of higher moments (often, the second moment $\left.\sum_{j} j^{2} \zeta_{j}\right)$ and (2.5) concerns the behavior of the first moment, the two definitions of gelation are usually equivalent.

Weak convergence of the Marcus-Lushnikov processes to either a Smoluchowski system or a modified Smoluchowski (Flory) system was explored in Jeon (1998), 
Norris (1999), Fournier and Giet (2004), and Fournier and Laurençot (2009). Specifically, it was shown in Fournier and Giet (2004) that if $\lim _{i \rightarrow \infty} \frac{K(i, j)}{i}=\ell(j)>0$, then the hydrodynamic limit of the Marcus-Lushnikov process with kernel $K(i, j)$ is the solution to the corresponding modified Smoluchowski (Flory) system. While, in Jeon (1998) and Norris (1999) it was established that $\lim _{i \rightarrow \infty} \frac{K(i, j)}{i}=0$ implies the hydrodynamic limit of the Marcus-Lushnikov process is the solution to the Smoluchowski system.

The question whether $T_{g e l}<\infty$ is the question of whether the gelation phenomenon occurs in a given system of Smoluchowski equations. The first mathematical proof of gelation was produced in McLeod (1962) for the multiplicative kernel. Historically, this happened around the time when the formation of a giant cluster in the Erdős-Rényi random graph model was proved by Erdős and Rényi (1960). The overlap in mathematical formulas obtained in the two papers, McLeod (1962) and Erdós and Rényi (1960), representing the two different branches of mathematics is quite remarkable. The work of finding a mathematically solid proof of gelation phenomenon for other conjectured gelling kernels began fifteen years later with the work of Lushnikov (1978). It continued with publications of Ziff (1980), Ernst et al. (1982), van Dongen and Ernst (1986), Jeon $(1998,1999)$, Escobedo et al. (2002), and many other mathematicians and mathematical physicists. In Spouge (1983), gelation is demonstrated numerically for the general bilinear kernel $K(i, j)=A+B(i+j)+C i j$. Aldous (1998) proved gelation for $K(i, j)=\frac{2(i j)^{\gamma}}{(i+j)^{\gamma}-i^{\gamma}-j^{\gamma}}$, where $\gamma \in(1,2)$. While $\gamma=2$ corresponds to the multiplicative kernel for which, as we know, gelation also occurs. Jeon (1999) proved that complete and instantaneous gelation occurs if $K(i, j) \geq i j \psi(i, j)$, where $\psi(i, j)$ is a function increasing in both variables, $i$ and $j$, such that $\sum_{j=1}^{\infty} \frac{1}{j \psi(i, j)}<\infty$ for all $i$. This includes $K(i, j)=(i j)^{\alpha}, \alpha>1$, as a primary example. Finally, Rezakhanlou (2013) lists sufficient conditions for each of the three modes of gelation, i.e., simple, instantaneous, and complete.

\section{The cross-multiplicative coalescent process}

In this section we analyze the cross-multiplicative coalescent process. We are motivated by the need to extend the theory and applications of coalescent processes to the particle system, described in the introduction, where not all pairs of particles interact with each other. Specifically, each particle may bond only with the particles of the opposite type.

For given $\alpha, \beta>0$, we consider two integer valued functions, $\alpha[n]=\alpha n+o(\sqrt{n})$ and $\beta[n]=\beta n+o(\sqrt{n})$. We will examine a coalescent process where the weight of each cluster is a two-dimensional (weight) vector $\mathbf{i}=\left[\begin{array}{l}i_{1} \\ i_{2}\end{array}\right]$. Here, $i_{1}, i_{2} \geq 0$ and $i_{1}+i_{2}>0$. Each cluster of weight $\mathbf{i}$ consists of $i_{1}$ particles of type $A$ and $i_{2}$ particles of type $B$. The coalescent process begins with $\alpha[n]+\beta[n]$ singletons, of which $\alpha[n]$ singletons are of weight $\left[\begin{array}{l}1 \\ 0\end{array}\right]$ and the other $\beta[n]$ singletons are of weight $\left[\begin{array}{l}0 \\ 1\end{array}\right]$. The coalescence kernel is defined by

$$
K(\mathbf{i}, \mathbf{j}):=i_{1} j_{2}+i_{2} j_{1}
$$


for any pair of clusters with weight vectors $\mathbf{i}=\left[\begin{array}{l}i_{1} \\ i_{2}\end{array}\right]$ and $\mathbf{j}=\left[\begin{array}{l}j_{1} \\ j_{2}\end{array}\right]$. Each pair of clusters of respective weights $\mathbf{i}$ and $\mathbf{j}$ would coalesce into a cluster of weight $\mathbf{i}+\mathbf{j}$ with rate $K(\mathbf{i}, \mathbf{j}) / n$. The last merger will create a cluster of weight $\left[\begin{array}{l}\alpha[n] \\ \beta[n]\end{array}\right]$. We will call this cross-multiplicative coalescent process, and the kernel $K(\mathbf{i}, \mathbf{j})$ defined in (3.1) will be referred to as the cross-multiplicative kernel.

3.1. Coagulation equations. Consider the Marcus-Lushnikov process $\mathbf{M} \mathbf{L}_{n}(t)$ that keeps track of cluster counts in the above defined cross-multiplicative coalescent process that begins with $\alpha[n]+\beta[n]$ singletons of the two types, $\alpha[n]$ of weight $\left[\begin{array}{l}1 \\ 0\end{array}\right]$ and $\beta[n]$ of weight $\left[\begin{array}{l}0 \\ 1\end{array}\right]$. Specifically, let $\zeta_{i_{1}, i_{2}}^{[n]}(t)$ denote the number of components of weight $\mathbf{i}=\left[\begin{array}{l}i_{1} \\ i_{2}\end{array}\right]$ at time $t$. Then $\mathbf{M L}_{n}(t)$ is the process with coordinates $\zeta_{i_{1}, i_{2}}^{[n]}(t)$, i.e.

$$
\mathbf{M L}_{n}(t)=\left(\zeta_{i_{1}, i_{2}}^{[n]}(t)\right)_{i_{1}, i_{2}}
$$

with the starting values $\zeta_{1,0}^{[n]}(0)=\alpha[n], \zeta_{0,1}^{[n]}(0)=\beta[n]$, and $\zeta_{i_{1}, i_{2}}^{[n]}(0)=0$ for all other pairs $\left(i_{1}, i_{2}\right)$.

The Smoluchowski coagulation equations for the Marcus-Lushnikov process $\mathbf{M L}_{n}(t)$ with cross-multiplicative kernel are written as follows:

$$
\begin{aligned}
\frac{d}{d t} \zeta_{i_{1}, i_{2}}(t)=-\zeta_{i_{1}, i_{2}}(t) & \sum_{j_{1}, j_{2}}\left(i_{1} j_{2}+i_{2} j_{1}\right) \zeta_{j_{1}, j_{2}}(t) \\
& +\frac{1}{2} \sum_{\substack{\ell_{1}, k_{1}: \ell_{1}+k_{1}=i_{1}, \ell_{2}, k_{2}: \ell_{2}+k_{2}=i_{2}}}\left(\ell_{1} k_{2}+\ell_{2} k_{1}\right) \zeta_{\ell_{1}, \ell_{2}}(t) \zeta_{k_{1}, k_{2}}(t)
\end{aligned}
$$

with the initial conditions $\zeta_{i_{1}, i_{2}}(0)=\alpha \delta_{1, i_{1}} \delta_{0, i_{2}}+\beta \delta_{0, i_{1}} \delta_{1, i_{2}}$.

A reduced system of differential equations corresponding to the above Smoluchowski coagulation equations (3.2) will be given in (3.5). It will take into account the mass conservation property of the above Marcus-Lushnikov process $\mathbf{M L}_{n}(t)$, and therefore will represent the smaller cluster dynamics over the whole time interval $[0, \infty)$.

First, we notice that here the initial total mass is $\sum_{i_{1}, i_{2}}\left(i_{1}+i_{2}\right) \zeta_{i_{1}, i_{2}}(0)=\alpha+\beta$. Moreover, the initial total 'left mass' (type $A$ ) is $\sum_{i_{1}, i_{2}} i_{1} \zeta_{i_{1}, i_{2}}(0)=\alpha$ and the initial total 'right mass' (type $B$ ) is $\sum_{i_{1}, i_{2}} i_{2} \zeta_{i_{1}, i_{2}}(0)=\beta$.

Next, we consider the rate of change for the total left mass and the total right mass, and use (3.2) to obtain

$$
\begin{aligned}
\frac{d}{d t} \sum_{i_{1}, i_{2}} i_{1} \zeta_{i_{1}, i_{2}}(t)=- & \sum_{i_{1}, i_{2}, j_{1}, j_{2}} i_{1}\left(i_{1} j_{2}+i_{2} j_{1}\right) \zeta_{i_{1}, i_{2}}(t) \zeta_{j_{1}, j_{2}}(t) \\
& +\frac{1}{2} \sum_{\ell_{1}, k_{1}, \ell_{2}, k_{2}}\left(\ell_{1}+k_{1}\right)\left(\ell_{1} k_{2}+\ell_{2} k_{1}\right) \zeta_{\ell_{1}, \ell_{2}}(t) \zeta_{k_{1}, k_{2}}(t)=0
\end{aligned}
$$


and

$$
\begin{aligned}
\frac{d}{d t} \sum_{i_{1}, i_{2}} i_{2} \zeta_{i_{1}, i_{2}}(t)=- & \sum_{i_{1}, i_{2}, j_{1}, j_{2}} i_{2}\left(i_{1} j_{2}+i_{2} j_{1}\right) \zeta_{i_{1}, i_{2}}(t) \zeta_{j_{1}, j_{2}}(t) \\
& +\frac{1}{2} \sum_{\ell_{1}, k_{1}, \ell_{2}, k_{2}}\left(\ell_{2}+k_{2}\right)\left(\ell_{1} k_{2}+\ell_{2} k_{1}\right) \zeta_{\ell_{1}, \ell_{2}}(t) \zeta_{k_{1}, k_{2}}(t)=0
\end{aligned}
$$

whenever $\sum_{i_{1}, i_{2}}\left(i_{1}+i_{2}\right)^{2} \zeta_{i_{1}, i_{2}}(t)$ converges.

Here, for $t<T_{\text {gel }}, \sum_{j_{1}, j_{2}} j_{1} \zeta_{j_{1}, j_{2}}(t)=\alpha$ and $\sum_{j_{1}, j_{2}} j_{2} \zeta_{j_{1}, j_{2}}(t)=\beta$. Therefore, for any $i_{1}$ and $i_{2}, \sum_{j_{1}, j_{2}}\left(i_{1} j_{2}+i_{2} j_{1}\right) \zeta_{j_{1}, j_{2}}(t)=\beta i_{1}+\alpha i_{2}$. Thus, we can consider the following modified Smoluchowski coagulation system of equations (Flory system):

$$
\frac{d}{d t} \zeta_{i_{1}, i_{2}}(t)=-\left(\beta i_{1}+\alpha i_{2}\right) \zeta_{i_{1}, i_{2}}(t)+\frac{1}{2} \sum_{\substack{\ell_{1}, k_{1}: \ell_{1}+k_{1}=i_{1}, \ell_{2}, k_{2}: \ell_{2}+k_{2}=i_{2}}}\left(\ell_{1} k_{2}+\ell_{2} k_{1}\right) \zeta_{\ell_{1}, \ell_{2}}(t) \zeta_{k_{1}, k_{2}}(t)
$$

with the initial conditions $\zeta_{i_{1}, i_{2}}(0)=\alpha \delta_{1, i_{1}} \delta_{0, i_{2}}+\beta \delta_{0, i_{1}} \delta_{1, i_{2}}$. Once again, the solutions of Smoluchowski coagulation system (3.2) and the above modified Smoluchowski coagulation system (3.5) will match up until $T_{g e l}$. Consequently, the solution (3.10) of the modified Smoluchowski system of equations (3.5) is used in Subsection 3.3 for establishing the finiteness of the gelation time and for finding its value, $T_{g e l}$.

In Subsection 5.2 we establish that the solution to the above modified Smoluchowski coagulation system (3.5) is the hydrodynamic limit of the MarcusLushnikov process $\mathbf{M L}_{n}(t)$ with cross-multiplicative kernel. Specifically, in equation (5.6), it is shown that

$$
\lim _{n \rightarrow \infty} \sup _{s \in[0, T]}\left|n^{-1} \zeta_{i_{1}, i_{2}}^{[n]}(s)-\zeta_{i_{1}, i_{2}}(s)\right|=0 \quad \text { a.s. }
$$

for any given $T>0$ and all $i_{1}, i_{2} \geq 1$, where $\zeta_{i_{1}, i_{2}}(t)$ solves the modified Smoluchowski coagulation system (3.5).

3.2. The unique solution of the modified Smoluchowski coagulation system. Next, we want to find the solution $\zeta_{i_{1}, i_{2}}(t)$ of the reduced system (3.5) for all $t \geq 0$. Here we observe that $\zeta_{1,0}(t)=\alpha e^{-\beta t}$ and $\zeta_{0,1}(t)=\beta e^{-\alpha t}$, and extend the approach of McLeod (1962) by considering the solutions of the following form

$$
\zeta_{i_{1}, i_{2}}(t)=\alpha^{i_{1}} \beta^{i_{2}} S_{i_{1}, i_{2}} e^{-\left(\beta i_{1}+\alpha i_{2}\right) t} t^{i_{1}+i_{2}-1}
$$

and plugging them into equation (3.5). After cancelations, we arrive with the following recursion

$$
\left(i_{1}+i_{2}-1\right) S_{i_{1}, i_{2}}=\frac{1}{2} \sum_{\substack{\ell_{1}, k_{1}: \ell_{1}+k_{1}=i_{1}, \ell_{2}, k_{2}: \ell_{2}+k_{2}=i_{2}}}\left(\ell_{1} k_{2}+\ell_{2} k_{1}\right) S_{\ell_{1}, \ell_{2}} S_{k_{1}, k_{2}}
$$

with initial conditions $S_{i, 0}=S_{0, i}=\delta_{1, i}$, and $S_{i_{1}, i_{2}}=S_{i_{2}, i_{1}}$.

In the next lemma we state the explicit solution to the recursion relation (3.7) which we prove using a generalization of Abel's binomial theorem. 
Lemma 3.1. The system of equations (3.7) with the initial conditions $S_{i, 0}=S_{0, i}=$ $\delta_{1, i}$ has the following unique solution

$$
S_{i_{1}, i_{2}}=\frac{i_{1}^{i_{2}-1} i_{2}^{i_{1}-1}}{i_{1} ! i_{2} !} .
$$

Note that the numerator $i_{1}^{i_{2}-1} i_{2}^{i_{1}-1}$ in (3.8) is the total number of spanning trees in $K_{i_{1}, i_{2}}$. See Austin (1960).

Proof: In Theorem 1.1(3) of Huang and Liu (2010), Abel's binomial theorem is generalized as follows:

$$
\begin{gathered}
\sum_{k_{1}=0}^{i_{1}} \sum_{k_{2}=0}^{i_{2}}\left(\begin{array}{l}
i_{1} \\
k_{1}
\end{array}\right)\left(\begin{array}{l}
i_{2} \\
k_{2}
\end{array}\right)\left(v+z i_{1}-z k_{1}\right)^{k_{2}-1}\left(-z\left(i_{1}-k_{1}\right)\right)^{i_{2}-k_{2}}\left(-z k_{2}\right)^{k_{1}}\left(u+z k_{2}\right)^{i_{1}-k_{1}-1} \\
=\frac{\left[u v-i_{1} i_{2} z^{2}\right] u^{i_{1}-1} v^{i_{2}-1}}{\left(v+i_{1} z\right)\left(u+i_{2} z\right)}
\end{gathered}
$$

Then, we use (3.9) with $z=-1$ to confirm our candidate solution satisfies (3.7) by plugging it into the right hand side of (3.7) as follows.

$$
\begin{aligned}
& \frac{1}{2} \sum_{\substack{\ell_{1}, k_{1}: \ell_{1}+k_{1}=i_{1}, \ell_{2}, k_{2}: \ell_{2}+k_{2}=i_{2}}}\left(\ell_{1} k_{2}+\ell_{2} k_{1}\right) S_{\ell_{1}, \ell_{2}} S_{k_{1}, k_{2}}=\sum_{\substack{\ell_{1}, k_{1}: \ell_{1}+k_{1}=i_{1}, \ell_{2}, k_{2}: \ell_{2}+k_{2}=i_{2}}} \ell_{1} k_{2} S_{\ell_{1}, \ell_{2}} S_{k_{1}, k_{2}} \\
& =\sum_{\substack{\ell_{1}, k_{1}: \ell_{1}+k_{1}=i_{1}, \ell_{2}, k_{2}: \ell_{2}+k_{2}=i_{2},\left(k_{1}, k_{2}\right),\left(\ell_{1}, \ell_{2}\right) \neq(0,0)}} \frac{\ell_{1}^{\ell_{2}} \ell_{2}^{\ell_{1}-1} k_{1}^{k_{2}-1} k_{2}^{k_{1}}}{\ell_{1} ! \ell_{2} ! k_{1} ! k_{2} !} \\
& =\frac{1}{i_{1} ! i_{2} !} \sum_{\substack{k_{1}: 0 \leq k_{1} \leq i_{1}, k_{2}: 0 \leq k_{2} \leq i_{2},\left(k_{1}, k_{2}\right) \neq(0,0),\left(i_{1}, i_{2}\right)}}\left(\begin{array}{c}
i_{1} \\
k_{1}
\end{array}\right)\left(\begin{array}{c}
i_{2} \\
k_{2}
\end{array}\right) k_{1}^{k_{2}-1}\left(i_{1}-k_{1}\right)^{i_{2}-k_{2}} k_{2}^{k_{1}}\left(i_{2}-k_{2}\right)^{i_{1}-k_{1}-1} \\
& =\frac{1}{i_{1} ! i_{2} !} \lim _{\substack{v \rightarrow i_{i} \\
u \rightarrow i_{2}}}\left\{\sum_{k_{1}=0}^{i_{1}} \sum_{k_{2}=0}^{i_{2}}\left(\begin{array}{l}
i_{1} \\
k_{1}
\end{array}\right)\left(\begin{array}{l}
i_{2} \\
k_{2}
\end{array}\right)\left(v-i_{1}+k_{1}\right)^{k_{2}-1}\left(i_{1}-k_{1}\right)^{i_{2}-k_{2}} k_{2}^{k_{1}}\left(u-k_{2}\right)^{i_{1}-k_{1}-1}\right. \\
& \left.-\frac{i_{1}^{i_{2}} u^{i_{1}-1}}{v-i_{1}}-\frac{i_{2}^{i_{1}} v^{i_{2}-1}}{u-i_{2}}\right\} \\
& =\frac{1}{i_{1} ! i_{2} !} \lim _{\substack{v \rightarrow i_{1} \\
u \rightarrow i_{2}}}\left\{\frac{\left[u v-i_{1} i_{2}\right] u^{i_{1}-1} v^{i_{2}-1}}{\left(v-i_{1}\right)\left(u-i_{2}\right)}-\frac{i_{1}^{i_{2}} u^{i_{1}-1}}{v-i_{1}}-\frac{i_{2}^{i_{1}} v^{i_{2}-1}}{u-i_{2}}\right\} \\
& =\frac{1}{i_{1} ! i_{2} !} \lim _{\substack{v \rightarrow i_{1} \\
u \rightarrow i_{2}}}\left\{\frac{i_{1} v^{i_{2}-1} u^{i_{1}-1}}{v-i_{1}}+\frac{u^{i_{1}} v^{i_{2}-1}}{u-i_{2}}-\frac{i_{1}^{i_{2}} u^{i_{1}-1}}{v-i_{1}}-\frac{i_{2}^{i_{1}} v^{i_{2}-1}}{u-i_{2}}\right\}
\end{aligned}
$$


Hence,

$$
\begin{aligned}
& \frac{1}{2} \sum_{\substack{\ell_{1}, k_{1}: \ell_{1}+k_{1}=i_{1} \\
\ell_{2}, k_{2}: \ell_{2}+k_{2}=i_{2}}}\left(\ell_{2} k_{2}+\ell_{2} k_{1}\right) S_{\ell_{1}, \ell_{2}} S_{k_{1}, k_{2}} \\
&=\frac{1}{i_{1} ! i_{2} !} \lim _{\substack{v \rightarrow i_{1} \\
u \rightarrow i_{2}}}\left\{i_{1} u^{i_{1}-1} \frac{v^{i_{2}-1}-i_{1}^{i_{2}-1}}{v-i_{1}}+v^{i_{2}-1} \frac{u^{i_{1}}-i_{2}^{i_{1}}}{u-i_{2}}\right\} \\
&=\frac{1}{i_{1} ! i_{2} !}\left(\left(i_{2}-1\right) \cdot i_{1}^{i_{2}-1} i_{2}^{i_{1}-1}+i_{1} \cdot i_{1}^{i_{2}-1} i_{2}^{i_{1}-1}\right) \\
&=\left(i_{1}+i_{2}-1\right) \frac{i_{1}^{i_{2}-1} i_{2}^{i_{1}-1}}{i_{1} ! i_{2} !}=\left(i_{1}+i_{2}-1\right) S_{i_{1}, i_{2}}
\end{aligned}
$$

thus completing the proof.

The solution of equations (3.5) follows from (3.6) and Lemma 3.1.

Theorem 3.2. The modified Smoluchowski coagulation system of equations (3.5) with the initial conditions $\zeta_{i_{1}, i_{2}}(0)=\alpha \delta_{1, i_{1}} \delta_{0, i_{2}}+\beta \delta_{0, i_{1}} \delta_{1, i_{2}}$ has the unique solution

$$
\zeta_{i_{1}, i_{2}}(t)=\frac{i_{1}^{i_{2}-1} i_{2}^{i_{1}-1} \alpha^{i_{1}} \beta^{i_{2}}}{i_{1} ! i_{2} !} e^{-\left(\beta i_{1}+\alpha i_{2}\right) t} t^{i_{1}+i_{2}-1} .
$$

3.3. Gelation in the cross-multiplicative coalescent process. Next, we prove the finiteness of the gelation time that, following the approach in (2.5), we define as

$$
T_{g e l}:=\inf \left\{t>0: \sum_{i_{1}, i_{2}}\left(i_{1}+i_{2}\right) \zeta_{i_{1}, i_{2}}(t)<\alpha+\beta\right\} .
$$

Let

$$
s(u, v):=\sum_{\left(i_{1}, i_{2}\right) \in \mathbb{Z}_{+}^{2} \backslash\{(0,0)\}} S_{i_{1}, i_{2}} u^{i_{1}} v^{i_{2}}=\sum_{\left(i_{1}, i_{2}\right) \in \mathbb{Z}_{+}^{2} \backslash\{(0,0)\}} \frac{i_{1}^{i_{2}-1} i_{2}^{i_{1}-1}}{i_{1} ! i_{2} !} u^{i_{1}} v^{i_{2}}
$$

be the generating function of $S_{i_{1}, i_{2}}$. The recurrence relation (3.7) implies

$$
u \frac{\partial s}{\partial u}+v \frac{\partial s}{\partial v}-s=u v \frac{\partial s}{\partial u} \frac{\partial s}{\partial v}
$$

with the initial conditions $\frac{\partial}{\partial u} s(0,1)=\frac{\partial}{\partial v} s(1,0)=1$.

Lemma 3.3. Consider the Smoluchowski coagulation system of equations (3.2) with the initial conditions $\zeta_{i_{1}, i_{2}}(0)=\alpha \delta_{1, i_{1}} \delta_{0, i_{2}}+\beta \delta_{0, i_{1}} \delta_{1, i_{2}}$. Then, a phase transition occurs at

$$
\inf \left\{t>0: \sum_{i_{1}, i_{2}}\left(i_{1}+i_{2}\right)^{2} \zeta_{i_{1}, i_{2}}(t)=\infty\right\}=\frac{1}{\sqrt{\alpha \beta}} .
$$

Note that the above phase transition corresponds to the gelation times as defined in (2.4).

Proof: We will follow the approach in Aldous $(1998,1999)$ and Ziff (1980). Let

$$
E(t):=\sum_{i_{1}, i_{2}} i_{1}^{2} \zeta_{i_{1}, i_{2}}(t), \quad F(t):=\sum_{i_{1}, i_{2}} i_{1} i_{2} \zeta_{i_{1}, i_{2}}(t), \quad \text { and } \quad G(t):=\sum_{i_{1}, i_{2}} i_{2}^{2} \zeta_{i_{1}, i_{2}}(t)
$$


denote all the second order moments of $\zeta_{i_{1}, i_{2}}(t)$. By differentiating as in (3.3) and (3.4), we obtain

$$
\frac{d}{d t} E(t)=2 E(t) F(t), \quad \frac{d}{d t} F(t)=E(t) G(t)+F^{2}(t), \quad \text { and } \quad \frac{d}{d t} G(t)=2 G(t) F(t)
$$

with the initial conditions $E(0)=\alpha, F(0)=0$, and $G(0)=\beta$. We require the finiteness of all third order moments when deriving the above differential equations for the second order moments. Here the first and the third equations yield $E(t)=$ $\frac{\alpha}{\beta} G(t)$. Hence the system reduces to

and therefore,

$$
\frac{d}{d t} E(t)=2 E(t) F(t) \quad \text { and } \quad \frac{d}{d t} F(t)=\frac{\beta}{\alpha} E^{2}(t)+F^{2}(t),
$$

$$
\frac{d}{d t}\left(\sqrt{\frac{\beta}{\alpha}} E(t)+F(t)\right)=\left(\sqrt{\frac{\beta}{\alpha}} E(t)+F(t)\right)^{2} .
$$

Thus,

$$
\sqrt{\frac{\beta}{\alpha}} E(t)+F(t)=\frac{1}{\frac{1}{\sqrt{\alpha \beta}}-t}
$$

for $t<\frac{1}{\sqrt{\alpha \beta}}$. The statement of the lemma follows from the fact that all functions obtained as all-order partial derivatives of the series (3.11) have the same domain of convergence.

For given $\alpha, \beta>0$ and $t>0$, define

$$
(x(t), y(t)):=\min \left\{(x, y): x e^{-y}=\alpha t e^{-\beta t}, y e^{-x}=\beta t e^{-\alpha t}\right\},
$$

where the minimum in one coordinate implies the minimum in another as $x$ and $y$ solving

$$
x e^{-y}=u \quad \text { and } \quad y e^{-x}=v
$$

for $u, v>0$ are mutually monotonous, e.g. $x=u e^{y}$.

Proposition 3.4. For given $u, v>0$, consider the system (3.14). Then, the following holds.

(i) Depending on the values of $u$ and $v$, the system (3.14) may have one, two, or no solutions.

(ii) If the system (3.14) has a unique solution, then the solution should satisfy $x y=1$.

(iii) If the system (3.14) has two solutions, then the smallest solution should satisfy $x y<1$, and the largest solution should satisfy $x y>1$.

Proof: First, observe that $x=u e^{v e^{x}}$, and statement (i) follows from the convexity of $u e^{v e^{x}}$.

Next, suppose $\left(x_{1}, y_{1}\right)$ and $\left(x_{2}, y_{2}\right)$ are two solutions of (3.14). Then

$$
x_{1} e^{-y_{1}}=x_{2} e^{-y_{2}} \quad \text { and } \quad y_{1} e^{-x_{1}}=y_{2} e^{-x_{2}} .
$$

We express $x_{2}$ in terms of $x_{1}$ and $y_{1}$, obtaining $x_{2}=x_{1} e^{y_{1}\left(e^{x_{2}-x_{1}}-1\right)}$. We notice that there is a unique solution $x=x_{1}$ of

$$
x=x_{1} e^{y_{1}\left(e^{x-x_{1}}-1\right)}
$$

if and only if $x=x_{1}$ is the root of $1=x y_{1} e^{x-x_{1}}$. This yields statement (ii). 
Finally, suppose there are two distinct solutions of (3.16), and $x_{2}>x_{1}$ (implying $\left.y_{2}>y_{1}\right)$. Then, there is a local extremum $x \in\left(x_{1}, x_{2}\right)$, satisfying $1=x y_{1} e^{x-x_{1}}>$ $x_{1} y_{1}$.

Similarly, suppose there are two distinct solutions of (3.16), and $x_{2}<x_{1}$ (implying $\left.y_{2}<y_{1}\right)$. Then, there is a local extremum $x \in\left(x_{2}, x_{1}\right)$, satisfying $1=$ $x y_{1} e^{x-x_{1}}<x_{1} y_{1}$. Hence, statement (iii).

Prop. 3.4 immediately yields the following corollary concerning the functions defined in (3.13).

Corollary 3.5. For given $\alpha, \beta>0$ and $t>0$, consider $(x(t), y(t))$ as defined in (3.13). Then,

- $x(t)=\alpha$ and $y(t)=\beta t$ for all $t \leq \frac{1}{\sqrt{\alpha \beta}}$;

- $x(t)<\alpha$ t and $y(t)<\beta$ for all $t>\frac{1}{\sqrt{\alpha \beta}}$.

Next, we prove the following lemma.

Lemma 3.6. Consider the solution $\zeta_{i_{1}, i_{2}}(t)$ of the modified Smoluchowski coagulation system of equations (3.5) with the initial conditions

$$
\zeta_{i_{1}, i_{2}}(0)=\alpha \delta_{1, i_{1}} \delta_{0, i_{2}}+\beta \delta_{0, i_{1}} \delta_{1, i_{2}},
$$

as found in Theorem 3.2. Then,

$$
\sum_{i_{1}, i_{2}} i_{1} \zeta_{i_{1}, i_{2}}(t)=\sum_{i_{1}, i_{2}} \frac{i_{1}^{i_{2}} i_{2}^{i_{1}-1} \alpha^{i_{1}} \beta^{i_{2}}}{i_{1} ! i_{2} !} e^{-\left(\beta i_{1}+\alpha i_{2}\right) t} t^{i_{1}+i_{2}-1}=\frac{x(t)}{t}
$$

and

$$
\sum_{i_{1}, i_{2}} i_{2} \zeta_{i_{1}, i_{2}}(t)=\sum_{i_{1}, i_{2}} \frac{i_{1}^{i_{2}-1} i_{2}^{i_{1}} \alpha^{i_{1}} \beta^{i_{2}}}{i_{1} ! i_{2} !} e^{-\left(\beta i_{1}+\alpha i_{2}\right) t} t^{i_{1}+i_{2}-1}=\frac{y(t)}{t},
$$

where $x(t)$ and $y(t)$ are the functions defined in (3.13).

Proof: Observe that

$$
\sum_{i_{1}, i_{2}} i_{1} \zeta_{i_{1}, i_{2}}(t)=\alpha t e^{-\beta t} \frac{\partial s}{\partial u}\left(\alpha t e^{-\beta t}, \beta t e^{-\alpha t}\right)
$$

and

$$
\sum_{i_{1}, i_{2}} i_{2} \zeta_{i_{1}, i_{2}}(t)=\beta t e^{-\alpha t} \frac{\partial s}{\partial v}\left(\alpha t e^{-\beta t}, \beta t e^{-\alpha t}\right)
$$

By (3.3), (3.4), and Lemma 3.3, we have

$$
\alpha t=\alpha t e^{-\beta t} \frac{\partial s}{\partial u}\left(\alpha t e^{-\beta t}, \beta t e^{-\alpha t}\right) \text { and } \beta t=\beta t e^{-\alpha t} \frac{\partial s}{\partial v}\left(\alpha t e^{-\beta t}, \beta t e^{-\alpha t}\right)
$$

$\forall \alpha, \beta>0$ and $\forall t<\frac{1}{\sqrt{\alpha \beta}}$. Now, since the function $s(u, v)$ does not depend on the values of $\alpha$ and $\beta$, (3.17) implies

$$
x=x e^{-y} \frac{\partial s}{\partial u}\left(x e^{-y}, y e^{-x}\right) \quad \text { and } \quad y=y e^{-x} \frac{\partial s}{\partial v}\left(x e^{-y}, y e^{-x}\right)
$$

for all $x y<1$. Hence, by Prop. 3.4 , we have

$$
x=u \frac{\partial s}{\partial u}(u, v) \quad \text { and } \quad y=v \frac{\partial s}{\partial v}(u, v)
$$


whenever $(x, y)$ is the smallest solution of (3.14). The equations

$x(t)=\alpha t e^{-\beta t} \frac{\partial s}{\partial u}\left(\alpha t e^{-\beta t}, \beta t e^{-\alpha t}\right)$ and $y(t)=\beta t e^{-\alpha t} \frac{\partial s}{\partial v}\left(\alpha t e^{-\beta t}, \beta t e^{-\alpha t}\right) \quad \forall t \geq 0$,

with $x(t)$ and $y(t)$ defined in (3.13), follow from Proposition 3.4.

Corollary 3.7. The cross-multiplicative kernel defined in (3.1) is a gelling kernel, and the gelation time corresponding to the Smoluchowski coagulation system of equations (3.2) with the initial conditions $\zeta_{i_{1}, i_{2}}(0)=\alpha \delta_{1, i_{1}} \delta_{0, i_{2}}+\beta \delta_{0, i_{1}} \delta_{1, i_{2}}$ equals

$$
T_{g e l}=\frac{1}{\sqrt{\alpha \beta}} \text {. }
$$

Proof: Lemma 3.6 and Proposition 3.4 imply that the mass of the system in (3.5) is conserved until $\frac{1}{\sqrt{\alpha \beta}}$, after which time it begins to dissipate, i.e.,

$$
\begin{cases}\sum_{i_{1}, i_{2}}\left(i_{1}+i_{2}\right) \zeta_{i_{1}, i_{2}}(t)=\alpha+\beta & \text { if } t \leq \frac{1}{\sqrt{\alpha \beta}} \\ \sum_{i_{1}, i_{2}}\left(i_{1}+i_{2}\right) \zeta_{i_{1}, i_{2}}(t)<\alpha+\beta & \text { if } t>\frac{1}{\sqrt{\alpha \beta}} .\end{cases}
$$

Recall that we considered two alternative definitions of gelation time in Subsection 2.1. Definition (2.4) would often describe the time of the explosion of a higher moment while definition (2.5) is based on the loss of total mass after gelation. Comparing Lemma 3.3 with Corollary 3.7, we confirm the equivalence of the two alternative definitions of the gelation time $T_{g e l}$ for the cross-multiplicative kernel, i.e.,

$\inf \left\{t>0: \sum_{i_{1}, i_{2}}\left(i_{1}+i_{2}\right)^{2} \zeta_{i_{1}, i_{2}}(t)=\infty\right\}=\inf \left\{t>0: \sum_{i_{1}, i_{2}}\left(i_{1}+i_{2}\right) \zeta_{i_{1}, i_{2}}(t)<\alpha+\beta\right\}$.

\section{Applications in minimal spanning trees}

In this section we demonstrate how the coagulation equations for the crossmultiplicative coalescent process and the weak convergence results of Section 5 are used for finding the asymptotic length of the minimal spanning tree on the complete bipartite graph $K_{\alpha[n], \beta[n]}$. The main result of this section Theorem 4.2 is proved using Marcus-Lushnikov processes and coagulation equations in Subsection 4.3.

We recall the following quote from Aldous (1998): It turns out that there is a large scientific literature relevant to the Marcus-Lushnikov process, mostly focusing on its deterministic approximation. Curiously, this literature has been largely ignored by random graph theorists. The broader goal of this section is in bridging the gap between the theory of the Smoluchowski coagulation equations for the Marcus-Lushnikov processes and the random graph theory. Here we concentrate on analyzing the length of the minimal spanning tree as the prime example that demonstrates the usefulness of the Marcus-Lushnikov processes and the coalescence theory in general for answering questions about random graphs. We recall that the asymptotic limit for the mean length of a minimal spanning tree on $K_{n}$ with independent uniform edge weights over [0,1] was derived in Frieze (1985). There, it is shown to be $\lim _{n \rightarrow \infty} E\left[L_{n}\right]=\zeta(3)=\sum_{k=1}^{\infty} \frac{1}{k^{3}}$. In Frieze and McDiarmid (1989), the 
mean length of the minimal spanning tree for the complete bipartite graph $K_{n, n}$ with independent edge weights distributed uniformly over $[0,1]$ was shown to have the asymptotic limit $\lim _{n \rightarrow \infty} E\left[L_{n}\right]=2 \zeta(3)$. In Beveridge et al. (1998), the minimal spanning tree problem was addressed for $d$-regular graphs. In this section, we will find the mean length of the minimal spanning tree in the case of a complete bipartite graph $K_{\alpha[n], \beta[n]}$ via a connection between the coalescence theory and the random graph theory. Note that $K_{\alpha[n], \beta[n]}$ is an irregular graph when $\alpha \neq \beta$.

4.1. Relation of Erdôs-Rényi process on $K_{\alpha[n], \beta[n]}$ to cross-multiplicative coalescent. Let $\alpha, \beta>0$ be given, and consider two integer valued functions, $\alpha[n]=\alpha n+o(\sqrt{n})$ and $\beta[n]=\beta n+o(\sqrt{n})$. Next, we introduce the Erdôs-Rényi random graph process on the bipartite graph $K_{\alpha[n], \beta[n]}$ with $\alpha[n]$ vertices on the left side and $\beta[n]$ vertices on the right side. In this random graph process on $K_{\alpha[n], \beta[n]}$, for each edge $e$ of $\alpha[n] \beta[n]=\alpha \beta n^{2}+o(n \sqrt{n})$ edges we have an associated random variable $U_{e}$, distributed uniformly on $[0,1]$. The random variables $\left\{U_{e}\right\}_{e}$ are assumed to be independent. For the "time" parameter $p \in[0,1]$, an edge $e$ is considered "open" if $U_{e} \leq p$. Erdős-Rényi random graph $G(n, p)$ will consist of all $n$ vertices and all open edges at time $p$.

In this Erdôs-Rényi random graph process, the probability of two components merging at a given time depends only on the number of edges that connect those two components. If connected component $C_{i}$ and $C_{j}$ have partition sizes $\left(i_{1}, i_{2}\right)$ and $\left(j_{1}, j_{2}\right)$ respectively, then there are $i_{1} j_{2}+i_{2} j_{1}$ edges which, when opened, would connect $C_{i}$ and $C_{j}$. Therefore, the cross-multiplicative coalescent process represents the cluster dynamics of the above Erdôs-Rényi random graph process on the bipartite graph $K_{\alpha[n], \beta[n]}$ under the time change $p=1-e^{-t / n}$. This coalescent process representation is obtained by letting each cluster connecting $i_{1}$ vertices on the left side of the bipartite graph with $i_{2}$ vertices on the right side of the bipartite graph be assigned a two-dimensional weight vector $\left[\begin{array}{l}i_{1} \\ i_{2}\end{array}\right]$. Then, the Marcus-Lushnikov process $\zeta_{i_{1}, i_{2}}^{[n]}(t)$ corresponding to the cross-multiplicative coalescent process will count the number of clusters with the weight vector $\left[\begin{array}{l}i_{1} \\ i_{2}\end{array}\right]$ at time $t$.

4.2. The length of the minimal spanning tree on $K_{\alpha[n], \beta[n]}$ via $\zeta_{i_{1}, i_{2}}(t)$. Consider the Erdôs-Rényi random graph model on a complete bipartite graph $K_{\alpha[n], \beta[n]}$. Let us interpret $U_{e}$ as the length of edge $e$. Then one can construct a minimal spanning tree on $K_{\alpha[n], \beta[n]}$. Let random variable $L_{n}$ denote the length of such minimal spanning tree. We want to represent the asymptotic limit of the mean value of $L_{n}$ via $\zeta_{i_{1}, i_{2}}(t)$.

For a random graph process $G(n, p)$ over $K_{\alpha[n], \beta[n]}$, Lemma 1 in Beveridge et al. (1998) implies

$$
E\left[L_{n}\right]=\int_{0}^{1} E[\kappa(G(n, p))] d p-1,
$$

where $\kappa(G(n, p))$ is the number of components in the random graph process $G(n, p)$ at time $p$. This will be used in Subsection 4.3 for proving the following theorem. 
Theorem 4.1. Let $\alpha, \beta>0$ and $L_{n}=L_{n}(\alpha, \beta)$ be the length of a minimal spanning tree on a complete bipartite graph $K_{\alpha[n], \beta[n]}$ with partitions of sizes

$$
\alpha[n]=\alpha n+o(\sqrt{n}) \text { and } \beta[n]=\beta n+o(\sqrt{n})
$$

and independent uniform edge weights over $[0,1]$. Then

$$
\lim _{n \rightarrow \infty} E\left[L_{n}\right]=\sum_{i_{1}, i_{2}}^{\infty} \int_{0}^{\infty} \zeta_{i_{1}, i_{2}}(t) d t .
$$

where $\zeta_{i_{1}, i_{2}}(t)$ indexed by $\mathbb{Z}_{+}^{2} \backslash\{(0,0)\}$ is the solution of the modified Smoluchowski coagulation system (3.5) with the initial conditions $\zeta_{i_{1}, i_{2}}(0)=\alpha \delta_{1, i_{1}} \delta_{0, i_{2}}+\beta \delta_{0, i_{1}} \delta_{1, i_{2}}$.

Observe that if we plug-in the solutions (3.6) of the reduced system of Smoluchowski coagulation equations (3.5) into the right hand side of (4.2), we get

$$
\begin{aligned}
\sum_{i_{1}, i_{2}}^{\infty} \int_{0}^{\infty} \zeta_{i_{1}, i_{2}}(t) d t & =\frac{\alpha}{\beta}+\frac{\beta}{\alpha}+\sum_{i_{1} \geq 1: i_{2} \geq 1} \alpha^{i_{1}} \beta^{i_{2}} S_{i_{1}, i_{2}} \int_{0}^{\infty} t^{i_{1}+i_{2}-1} e^{-\left(\beta i_{1}+\alpha i_{2}\right) t} d t \\
& =\frac{\alpha}{\beta}+\frac{\beta}{\alpha}+\sum_{i_{1} \geq 1: i_{2} \geq 1} \frac{\alpha^{i_{1}} \beta^{i_{2}} S_{i_{1}, i_{2}}}{\left(\beta i_{1}+\alpha i_{2}\right)^{i_{1}+i_{2}}}\left(i_{1}+i_{2}-1\right) ! \\
& =\gamma+\frac{1}{\gamma}+\sum_{i_{1} \geq 1: i_{2} \geq 1} \frac{\gamma^{i_{1}} S_{i_{1}, i_{2}}}{\left(i_{1}+\gamma i_{2}\right)^{i_{1}+i_{2}}}\left(i_{1}+i_{2}-1\right) !
\end{aligned}
$$

with $\gamma=\frac{\alpha}{\beta}$.

Next, by combining Lemma 3.1 with (4.3) we obtained the following important theorem.

Theorem 4.2. Let $\alpha, \beta>0, \gamma=\alpha / \beta$, and $L_{n}=L_{n}(\alpha, \beta)$ be the length of $a$ minimal spanning tree on a complete bipartite graph $K_{\alpha[n], \beta[n]}$ with partitions of sizes

$$
\alpha[n]=\alpha n+o(\sqrt{n}) \text { and } \beta[n]=\beta n+o(\sqrt{n})
$$

and independent uniform edge weights over $[0,1]$. Then the limiting mean length of the minimal spanning tree is

$$
\lim _{n \rightarrow \infty} E\left[L_{n}\right]=\gamma+\frac{1}{\gamma}+\sum_{i_{1} \geq 1 ; i_{2} \geq 1} \frac{\left(i_{1}+i_{2}-1\right) !}{i_{1} ! i_{2} !} \frac{\gamma^{i_{1}} i_{1}^{i_{2}-1} i_{2}^{i_{1}-1}}{\left(i_{1}+\gamma i_{2}\right)^{i_{1}+i_{2}}} .
$$

Theorem 4.2 is consistent with Frieze and McDiarmid (1989), where it was shown that for $\alpha=\beta, \lim _{n \rightarrow \infty} E\left[L_{n}\right]=2 \zeta(3)$. Indeed, we have the following Corollary reproducing the results in Frieze and McDiarmid (1989). Observe however that for $\alpha \neq \beta$ the bipartite graph is irregular and the results in Frieze and McDiarmid (1989) no longer apply.

Corollary 4.3. If $\gamma=1$, then

$$
\lim _{n \rightarrow \infty} E\left[L_{n}\right]=2 \zeta(3) .
$$

Proof: Abel's binomial theorem states that

$$
\sum_{k=0}^{n}\left(\begin{array}{l}
n \\
k
\end{array}\right)(x-k z)^{k-1}(y+k z)^{n-k}=x^{-1}(x+y)^{n} .
$$


See Riordan (1968); Comtet (1974). Substituting $x=n z \neq 0, y=0$, and $i=n-k$, we obtain

and therefore,

$$
\sum_{i=0}^{n}\left(\begin{array}{c}
n \\
i
\end{array}\right) i^{n-i-1}(n-i)^{i}=n^{n-1}
$$

$$
\sum_{i_{1}, i_{2}: i_{1}+i_{2}=n} i_{1} S_{i_{1}, i_{2}}=\sum_{i_{1}, i_{2}: i_{1}+i_{2}=n} i_{1} \frac{i_{1}^{i_{2}-1} i_{2}^{i_{1}-1}}{i_{1} ! i_{2} !}=\frac{n^{n-1}}{n !} .
$$

Hence,

$$
n \cdot \sum_{i_{1}, i_{2}: i_{1}+i_{2}=n} S_{i_{1}, i_{2}}=\sum_{i_{1}, i_{2}: i_{1}+i_{2}=n}\left(i_{1}+i_{2}\right) S_{i_{1}, i_{2}}=2 \cdot \sum_{i_{1}, i_{2}: i_{1}+i_{2}=n} i_{1} S_{i_{1}, i_{2}}=2 \frac{n^{n-1}}{n !}
$$

and

$$
\sum_{i_{1}, i_{2}: i_{1}+i_{2}=n} S_{i_{1}, i_{2}}=2 \frac{n^{n-2}}{n !}
$$

Plugging the above into (4.3) with $\gamma=1$, we obtain

$$
\begin{aligned}
\lim _{n \rightarrow \infty} E\left[L_{n}\right] & =2+\sum_{i_{1} \geq 1: i_{2} \geq 1} \frac{S_{i_{1}, i_{2}}}{\left(i_{1}+i_{2}\right)^{i_{1}+i_{2}}}\left(i_{1}+i_{2}-1\right) ! \\
& =2+\sum_{n=2}^{\infty}\left(\sum_{i_{1}, i_{2}: i_{1}+i_{2}=n} \frac{S_{i_{1}, i_{2}}}{n^{n}}\right)(n-1) ! \\
& =2+\sum_{n=2}^{\infty} 2 \frac{n^{n-2}}{n !} \cdot \frac{1}{n^{n}}(n-1) ! \\
& =2+\sum_{n=2}^{\infty} \frac{2}{n^{3}}=2 \zeta(3) .
\end{aligned}
$$

Thus confirming the results in Frieze and McDiarmid (1989).

4.3. Proof of Theorem 4.1. Let us give a rigorous proof of Theorem 4.1.

Proof: Observe that

$$
\lim _{t \rightarrow \infty} \sum_{i_{1}, i_{2}} i_{1} \zeta_{i_{1}, i_{2}}(t)=0 \quad \text { and } \quad \lim _{t \rightarrow \infty} \sum_{i_{1}, i_{2}} i_{2} \zeta_{i_{1}, i_{2}}(t)=0 .
$$

Indeed, by plugging in $\zeta_{i_{1}, i_{2}}(t)$ as in (3.10), we obtain

$\frac{d}{d t} \sum_{i_{1}, i_{2}} i_{1} \zeta_{i_{1}, i_{2}}(t)=\sum_{i_{1}, i_{2}} i_{1} \zeta_{i_{1}, i_{2}}(t)\left(\frac{i_{1}+i_{2}-1}{t}-\left(\beta i_{1}+\alpha i_{2}\right)\right) \leq-(\alpha \wedge \beta) \sum_{i_{1}, i_{2}} i_{1} \zeta_{i_{1}, i_{2}}(t)$ for $t>\frac{1}{\alpha \wedge \beta}$. Thus, $\sum_{i_{1}, i_{2}} i_{1} \zeta_{i_{1}, i_{2}}(t)$, and similarly $\sum_{i_{1}, i_{2}} i_{2} \zeta_{i_{1}, i_{2}}(t)$, would decrease to zero exponentially fast when $t>\frac{1}{\alpha \wedge \beta}$.

Now, having established (4.5), for any given $\epsilon \in(0,1 / 4)$, we can fix $T \gg T_{\text {gel }}$ so large that

$$
\sum_{i_{1}, i_{2}} i_{1} \zeta_{i_{1}, i_{2}}(t) \leq \frac{\alpha \epsilon}{2} \quad \text { and } \quad \sum_{i_{1}, i_{2}} i_{2} \zeta_{i_{1}, i_{2}}(t) \leq \frac{\beta \epsilon}{2} .
$$

Notice that the above inequalities (4.6) ties $T$ to $\epsilon$. 
Fix integers $K_{1}>0$ and $K_{2}>0$, and let

$$
R:=R\left(K_{1}, K_{2}\right)=\left\{1,2, \ldots, K_{1}\right\} \times\left\{1,2, \ldots, K_{2}\right\} .
$$

By the equation (5.7) in Subsection 5.2 we have

$$
\lim _{n \rightarrow \infty} \sup _{s \in[0, T]}\left|n^{-1} \sum_{R} \zeta_{i_{1}, i_{2}}^{[n]}(s)-\sum_{R} \zeta_{i_{1}, i_{2}}(s)\right|=0 \quad \text { a.s. }
$$

Thus, the probability of the complement of the event

$$
Q_{R, T, n}^{\epsilon}:=\left\{\sum_{\mathbf{i} \in R} i_{1} \frac{\zeta_{i_{1}, i_{2}}^{[n]}(T)}{n} \leq \frac{3}{4} \alpha \epsilon \quad \text { and } \quad \sum_{\mathbf{i} \in R} i_{2} \frac{\zeta_{i_{1}, i_{2}}^{[n]}(T)}{n} \leq \frac{3}{4} \beta \epsilon\right\}
$$

is decreasing to zero as $n \rightarrow \infty$. Moreover,

$$
q_{R, T}^{\epsilon}(n):=P\left(\overline{Q_{R, T, n}^{\epsilon}}\right)=O\left(n^{-2}\right)
$$

by Proposition 5.3 in Subsection 5.3 since

$$
\lim _{n \rightarrow \infty} \sqrt{n}\left(\sum_{\mathbf{i} \in R} i_{1} \frac{\zeta_{i_{1}, i_{2}}^{[n]}(0)}{n}-\sum_{\mathbf{i} \in R} i_{1} \zeta_{i_{1}, i_{2}}(0)\right)=\lim _{n \rightarrow \infty} \sqrt{n}(\alpha[n] / n-\alpha)=0
$$

and

$$
\lim _{n \rightarrow \infty} \sqrt{n}\left(\sum_{\mathbf{i} \in R} i_{2} \frac{\zeta_{i_{1}, i_{2}}^{[n]}(0)}{n}-\sum_{\mathbf{i} \in R} i_{2} \zeta_{i_{1}, i_{2}}(0)\right)=\lim _{n \rightarrow \infty} \sqrt{n}(\beta[n] / n-\beta)=0 .
$$

We know from (4.1) that

$$
\lim _{n \rightarrow \infty} E\left[L_{n}\right]=\lim _{n \rightarrow \infty} \int_{0}^{1} E[\kappa(G(n, p))] d p-1=\lim _{n \rightarrow \infty} \int_{0}^{\infty} \sum_{i_{1}, i_{2}} \frac{E\left[\zeta_{i_{1}, i_{2}}^{[n]}(t)\right]}{n} e^{-t / n} d t-1
$$

provided the latter limit exists.

We will split $\int_{0}^{\infty} \sum_{i_{1}, i_{2}} \frac{E\left[\zeta_{i_{1}, i_{2}}^{[n]}(t)\right]}{n} e^{-t / n} d t$ as follows.

$$
\begin{aligned}
\int_{0}^{\infty} \sum_{i_{1}, i_{2}} \frac{E\left[\zeta_{i_{1}, i_{2}}^{[n]}(t)\right]}{n} e^{-t / n} d t & =\int_{0}^{T} \sum_{\mathbf{i} \in R} \frac{E\left[\zeta_{i_{1}, i_{2}}^{[n]}(t)\right]}{n} e^{-t / n} d t \\
& +\int_{0}^{T} \sum_{\mathbf{i} \notin R} \frac{E\left[\zeta_{i_{1}, i_{2}}^{[n]}(t)\right]}{n} e^{-t / n} d t \\
& +\left(1-q_{R, T}^{\epsilon}(n)\right) \int_{T}^{\infty} \sum_{\mathbf{i} \in R} \frac{E\left[\zeta_{i_{1}, i_{2}}^{[n]}(t) \mid Q_{R, T, n}^{\epsilon}\right]}{n} e^{-t / n} d t \\
& +\left(1-q_{R, T}^{\epsilon}(n)\right) \int_{T}^{\infty} \frac{E\left[\zeta_{i}^{[n]}(t) \mid Q_{R, T, n}^{\epsilon}\right]}{n} e^{-t / n} d t \\
& +q_{R, T}^{\epsilon}(n) \int_{T}^{\infty} \sum_{i_{1}, i_{2}} \frac{E\left[\zeta_{i_{1}, i_{2}}^{[n]}(t) \mid \overline{Q_{R, T, n}}\right]}{n} e^{-t / n} d t
\end{aligned}
$$


Next, we estimate the terms $\mathbf{I}-\mathbf{V}$ in (4.8).

Term I. As we establish in (5.6) of Section 5,

$$
\lim _{n \rightarrow \infty} \sup _{s \in[0, T]}\left|n^{-1} \zeta_{i_{1}, i_{2}}^{[n]}(s)-\zeta_{i_{1}, i_{2}}(s)\right|=0 \text { a.s. }
$$

on $[0, T]$ for all $\mathbf{i}=\left[\begin{array}{l}i_{1} \\ i_{2}\end{array}\right] \in R$. Therefore,

$$
\lim _{n \rightarrow \infty} \int_{0}^{T} \sum_{\mathbf{i} \in R} \frac{E\left[\zeta_{i_{1}, i_{2}}^{[n]}(t)\right]}{n} e^{-t / n} d t=\sum_{\mathbf{i} \in R} \int_{0}^{T} \zeta_{i_{1}, i_{2}}(t) d t
$$

Term II. Observe that,

$$
\begin{aligned}
\sum_{\mathbf{i} \notin R} \frac{\zeta_{i_{1}, i_{2}}^{[n]}(t)}{n} & \leq \frac{1}{n} \sum_{i_{1}>K_{1}} \sum_{i_{2}} \zeta_{i_{1}, i_{2}}^{[n]}(t)+\frac{1}{n} \sum_{i_{1}} \sum_{i_{2}>K_{2}} \zeta_{i_{1}, i_{2}}^{[n]}(t) \\
& \leq \frac{1}{K_{1} n} \sum_{i_{1}>K_{1}} \sum_{i_{2}} i_{1} \zeta_{i_{1}, i_{2}}^{[n]}(t)+\frac{1}{n K_{2}} \sum_{i_{1}} \sum_{i_{2}>K_{2}} i_{2} \zeta_{i_{1}, i_{2}}^{[n]}(t) \\
& \leq \frac{\alpha[n]}{K_{1} n}+\frac{\beta[n]}{n K_{2}} \leq 2 \frac{\alpha}{K_{1}}+2 \frac{\beta}{K_{2}}
\end{aligned}
$$

for all $n$ large enough. Thus,

$$
\int_{0}^{T} \sum_{\mathbf{i} \notin R} \frac{E\left[\zeta_{i_{1}, i_{2}}^{[n]}(t)\right]}{n} e^{-t / n} d t=O\left(\frac{T}{K_{1}}\right)+O\left(\frac{T}{K_{2}}\right) .
$$

Term III. We define the $R$-gel to be the collection of all clusters whose mass vector is not in $R$. Let

$$
M_{R g e l}(t)=\left[\begin{array}{l}
m_{1}(t) \\
m_{2}(t)
\end{array}\right]
$$

denote the total mass vector of all clusters in the $R$-gel at time $t \geq 0$.

Now, conditioning on the event $Q_{R, T, n}^{\epsilon}$, we have $m_{1}(t) \geq \alpha(1-\epsilon) n$ and $m_{2}(t) \geq$ $\beta(1-\epsilon) n$ for all $t \geq T$, and $n$ large enough. Thus each cluster in $R$ will be gravitating toward the $R$-gel with the rate of at least $(\alpha \wedge \beta)(1-\epsilon)$. Consider a cluster in $R$ at time $T$. Let $T+L$ be the time it becomes a part of the $R$-gel. Then, its contribution to the integral $\int_{T}^{\infty} \sum_{\mathbf{i} \in R} \frac{E\left[\zeta_{i_{1}, i_{2}}^{[n]}(t) \mid Q_{R, T, n}^{\epsilon}\right]}{n} e^{-t / n} d t$ is at most

$$
\int_{T}^{\infty} \frac{E\left[\mathbf{1}_{[T, T+L]}(t) \mid Q_{R, T, n}^{\epsilon}\right]}{n} e^{-t / n} d t \leq \frac{E\left[L \mid Q_{R, T, n}^{\epsilon}\right]}{n} e^{-T / n} \leq \frac{1}{(\alpha \wedge \beta)(1-\epsilon) n} .
$$

The number of clusters in $R$ at time $t \geq T$ is

$$
\sum_{\mathbf{i} \in R} \zeta_{i_{1}, i_{2}}^{[n]}(t) \leq \sum_{\mathbf{i} \in R}\left(i_{1}+i_{2}\right) \zeta_{i_{1}, i_{2}}^{[n]}(t) \leq(\alpha+\beta) \epsilon n .
$$


Therefore,

$$
\int_{T}^{\infty} \sum_{\mathbf{i} \in R} \frac{E\left[\zeta_{i_{1}, i_{2}}^{[n]}(t) \mid Q_{R, T, n}^{\epsilon}\right]}{n} e^{-t / n} d t \leq \frac{(\alpha+\beta) \epsilon n}{(\alpha \wedge \beta)(1-\epsilon) n}=\frac{2 \epsilon}{1-\epsilon}<3 \epsilon .
$$

Term IV. We let $\mathcal{C}=\left\{C_{1}, C_{2}, C_{3}, \ldots, C_{M}\right\}$ denote the set of all clusters whose mass vectors ever exceeded $K_{1}$ in the first coordinate and/or ever exceeded $K_{2}$ in the second coordinate in the history of the process $\mathbf{M L}_{n}(t)$, i.e., all clusters that were ever a part of $R$-gel. The number of clusters in $\mathcal{C}$ is less than $\alpha[n] / K_{1}+\beta[n] / K_{2}$. For each $C_{i}$, the emergence time $a_{i}$ is the time of a merger of a pair of clusters in $R$, resulting in appearance of a new cluster $C_{i}$ in $R$-gel. We enumerate these clusters in the order they emerge.

Let $M_{i}(t)=\left[\begin{array}{l}m_{1, i}(t) \\ m_{2, i}(t)\end{array}\right]$ denote the mass vector of cluster $C_{i}$ at time $t$. Consider a pair of clusters, $C_{i}$ and $C_{j}$, coexisting in the $R$-gel at time $t$, such that $m_{1, i}, m_{1, j}<$ $\alpha n / 2$ and $m_{2, i}, m_{2, j}<\beta n / 2$. We split their merger rate into two by saying that $C_{i}$ absorbs $C_{j}$ with rate $\frac{1}{2 n}\left(m_{1, i}(t) m_{2, j}(t)+m_{2, i}(t) m_{1, j}(t)\right)$, and $C_{j}$ absorbs $C_{i}$ with rate $\frac{1}{2 n}\left(m_{1, i}(t) m_{2, j}(t)+m_{2, i}(t) m_{1, j}(t)\right)$.

There is a finite stopping time

$$
t^{*}=\min \left\{t \geq 0: \exists C_{i} \in \mathcal{C} \text { with } m_{1, i}(t) \geq \alpha n / 2 \text { or } m_{2, i}(t) \geq \beta n / 2\right\}
$$

when a cluster $C_{i^{*}}$ has its mass vector satisfying either $m_{1, i^{*}}\left(t^{*}\right) \geq \alpha n / 2$ or $m_{2, i^{*}}\left(t^{*}\right) \geq \beta n / 2$. After time $t^{*}$ the rules of interactions of cluster $C_{i^{*}}$ with the other clusters in $\mathcal{C}$ change as follows. For $t>t^{*}, C_{i^{*}}$ absorbs $C_{j}$ with rate $\frac{1}{n}\left(m_{1, i^{*}}(t) m_{2, j}(t)+m_{2, i^{*}}(t) m_{1, j}(t)\right)$, while $C_{i^{*}}$ itself cannot be absorbed by any other cluster in $\mathcal{C}$.

Let $b_{i}$ denote the time when cluster $C_{i}$ is absorbed by another cluster in collection $\mathcal{C}$. Naturally, there will be only one survivor $C_{i^{*}}$ with $b_{i^{*}}=\infty$. Let $J_{i}=\left[a_{i}, b_{i}\right) \cap$ $[T, \infty)$ denote the lifespan of cluster $C_{i}$. Note that a cluster $C_{i}$ from the collection $\mathcal{C}$ existing at time $t \in\left[a_{i}, b_{i}\right)$ is absorbed into one of the clusters in the $R$-gel with the total instantaneous rate of

$$
\lambda_{i}(t) \geq \frac{1}{2 n}\left(m_{1, i}(t)\left(m_{2}(t)-m_{2, i}(t)\right)+m_{2, i}(t)\left(m_{1}(t)-m_{1, i}(t)\right)\right),
$$

where $m_{1}(t)$ and $m_{2}(t)$ are as defined in (4.9). Conditioning on the event $Q_{R, T, n}^{\epsilon}$ defined in (4.7), we have that if $m_{1, i}(t)<\alpha n / 2$ and $m_{2, i}(t)<\beta n / 2$ for $t \in J_{i}$, then the rate of absorption of $C_{i}$ into the $R$-gel is

$$
\begin{aligned}
\lambda_{i}(t) & \geq \frac{1}{2 n} m_{1, i}(t) \beta\left((1-\epsilon) n-\frac{1}{2} n\right)+\frac{1}{2 n} m_{2, i}(t) \alpha\left((1-\epsilon) n-\frac{1}{2} n\right) \\
& \geq \frac{1}{2 n} m_{1, i}(t) \beta\left(\frac{3}{4} n-\frac{1}{2} n\right)+\frac{1}{2 n} m_{2, i}(t) \alpha\left(\frac{3}{4} n-\frac{1}{2} n\right) \\
& \geq \frac{m_{1, i}(t) \beta+m_{2, i}(t) \alpha}{8}>\frac{K_{1} \beta+K_{2} \alpha}{8} .
\end{aligned}
$$

Next,

$$
\int_{T}^{\infty} \sum_{\mathbf{i} \notin R} \frac{E\left[\zeta_{i_{1}, i_{2}}^{[n]}(t) \mid Q_{R, T, n}^{\epsilon}\right]}{n} e^{-t / n} d t=\int_{T}^{\infty} \frac{1}{n} e^{-t / n} d t+\mathcal{E}
$$


where $\int_{T}^{\infty} \frac{1}{n} e^{-t / n} d t$ is due to the event $Q_{R, T, n}^{\epsilon}$ which guarantees the existence of at least one component from $\mathcal{C}$ in the $R$-gel for all $t \in[T, \infty)$ and the second term $\mathcal{E}$ is responsible for all the times $t \geq T$ when the number of clusters in the $R$-gel is greater than one. The term $\mathcal{E}$ is bounded as follows

$$
\mathcal{E} \leq \int_{T}^{\infty} \frac{E\left[\sum_{i: i \neq i^{*}} \boldsymbol{1}_{J_{i}}(t) \mid Q_{R, T, n}^{\epsilon}\right]}{n} e^{-t / n} d t .
$$

Now, each cluster $C_{i}$ is gravitating towards the rest of the $R$-gel with the rate of at least $\frac{K_{1} \beta+K_{2} \alpha}{8}$. Thus, for each $i \neq i^{*}$,

$$
\int_{T}^{\infty} \frac{E\left[\mathbf{1}_{J_{i}}(t) \mid Q_{R, T, n}^{\epsilon}\right]}{n} e^{-t / n} d t \leq \frac{E\left[\left|J_{i}\right| \mid Q_{R, T, n}^{\epsilon}\right]}{n} e^{-\frac{T}{n}} \leq \frac{8}{n\left(K_{1} \beta+K_{2} \alpha\right)} .
$$

Hence, since the cardinality of set $\mathcal{C}$ is $M<\alpha[n] / K_{1}+\beta[n] / K_{2}$,

$$
\mathcal{E}<\left(\alpha[n] / K_{1}+\beta[n] / K_{2}\right) \cdot \frac{8}{n\left(K_{1} \beta+K_{2} \alpha\right)}=\frac{8\left(\alpha / K_{1}+\beta / K_{2}\right)}{K_{1} \beta+K_{2} \alpha}+o(1),
$$

and from (4.10), we obtain

$$
\int_{T}^{\infty} \sum_{\mathbf{i} \notin R} \frac{E\left[\zeta_{i_{1}, i_{2}}^{[n]}(t) \mid Q_{R, T, n}^{\epsilon}\right]}{n} e^{-t / n} d t=1+O\left(K_{1}^{-2}\right)+O\left(K_{2}^{-2}\right)+O\left(\frac{T}{n}\right)+o(1) \text { as } n \rightarrow \infty .
$$

\section{Term V. Here}

$$
\begin{aligned}
q_{R, T}^{\epsilon}(n) \int_{T}^{\infty} \sum_{i_{1}, i_{2}} \frac{E\left[\zeta_{i_{1}, i_{2}}^{[n]}(t) \mid \overline{Q_{R, T, n}^{\epsilon}}\right]}{n} e^{-t / n} d t & \leq q_{R, T}^{\epsilon}(n) \int_{T}^{\infty} \frac{\alpha[n]+\beta[n]}{n} e^{-t / n} d t \\
& \leq(\alpha[n]+\beta[n]) q_{R, T}^{\epsilon}(n)=O\left(n^{-1}\right)
\end{aligned}
$$

as $q_{R, T}^{\epsilon}(n)=O\left(n^{-2}\right)$.

Finally, by putting together the analysis in Terms I-V in the equation (4.8), we obtain for a given fixed $\epsilon \in(0,1 / 4)$, sufficiently large fixed $T \gg T_{g e l}$ satisfying (4.6), and arbitrarily large $K_{1}$ and $K_{2}$,

$$
\begin{aligned}
\int_{0}^{\infty} \sum_{i_{1}, i_{2}} \frac{E\left[\zeta_{i_{1}, i_{2}}^{[n]}(t)\right]}{n} e^{-t / n} d t= & \sum_{\mathbf{i} \in R\left(K_{1}, K_{2}\right)} \int_{0}^{T} \zeta_{i_{1}, i_{2}}(t) d t+1+O\left(\frac{T}{K_{1}}\right)+O\left(\frac{T}{K_{2}}\right) \\
& +O\left(K_{1}^{-2}\right)+O\left(K_{2}^{-2}\right)+O(\epsilon)+O\left(\frac{T}{n}\right)+O\left(n^{-1}\right)
\end{aligned}
$$

which when we increase $n$ to infinity will yield

$$
\lim _{n \rightarrow \infty} \int_{0}^{\infty} \sum_{i_{1}, i_{2}} \frac{E\left[\zeta_{i_{1}, i_{2}}^{[n]}(t)\right]}{n} e^{-t / n} d t=\sum_{i_{1}, i_{2}} \int_{0}^{\infty} \zeta_{i_{1}, i_{2}}(t) d t+1 .
$$




\section{Hydrodynamic limits for Marcus-Lushnikov processes}

In Kurtz (1981) and Ethier and Kurtz (1986), a certain class of Markov processes, called density dependent population processes, was considered. These are jump Markov processes which depend on a certain parameter $n$ which can be interpreted depending on the context of a model. Usually it represents the population size. Many coalescent processes can be restated as a case of density dependent population processes if all cluster weights are integers. There, the total mass $n$ is the parameter representing the population size. Specifically, we may assume that the coalescent process starts with $n$ clusters of unit mass each (aka singletons). In Kurtz (1981) and in Chapter 11 of Ethier and Kurtz (1986), the law of large numbers and the central limit theorems were established for such density dependent population processes as $n \rightarrow \infty$. In this section we will adopt these weak limit laws for the cross-multiplicative coalescent process.

5.1. Density dependent population processes. We first formulate the framework for the convergence result of Kurtz as stated in Theorem 2.1 in Chapter 11 of Ethier and Kurtz (1986), and earlier, in Theorem 8.1 of Kurtz (1981). There, the density dependent population processes are defined as continuous time Markov processes with state spaces in $\mathbb{Z}^{d}$, and transition intensities represented as follows

$$
q^{(n)}(k, k+\ell)=n\left[\beta_{\ell}\left(\frac{k}{n}\right)+O\left(\frac{1}{n}\right)\right],
$$

where $\ell, k \in \mathbb{Z}^{d}$, and $\beta_{\ell}$ is a given collection of rate functions.

In Section 5.1 of Aldous (1999), Aldous observes that the results from Chapter 11 of Ethier and Kurtz (1986) can be used to prove the weak convergence of a MarcusLushnikov process to the solutions of Smoluchowski system of equations in the case when the Marcus-Lushnikov process can be formulated as a finite dimensional density dependent population process. Specifically, the Marcus-Lushnikov processes corresponding to the multiplicative and Kingman coalescent with the monodisperse initial conditions ( $n$ singletons) can be represented as finite dimensional density dependent population processes defined above.

Define $F(x)=\sum_{\ell} \ell \beta_{\ell}(x)$. Then, Theorem 2.1 in Chapter 11 of Ethier and Kurtz (1986), same as Theorem 8.1 in Kurtz (1981), states the following law of large numbers. Let $\hat{X}_{n}(t)$ be the Markov process with the intensities $q^{(n)}(k, k+\ell)$ given in (5.1), and let $X_{n}(t)=n^{-1} \hat{X}_{n}(t)$. Finally, let $|x|=\sqrt{\sum x_{i}^{2}}$ denote the Euclidean norm in $\mathbb{R}^{d}$.

Theorem 5.1. Suppose for all compact $\mathcal{K} \subset \mathbb{R}^{d}$,

$$
\sum_{\ell}|\ell| \sup _{x \in \mathcal{K}} \beta_{\ell}(\bar{x})<\infty,
$$

and there exists $M_{\mathcal{K}}>0$ such that

$$
|F(x)-F(y)| \leq M_{\mathcal{K}}|x-y|, \quad \text { for all } x, y \in \mathcal{K} .
$$

Suppose $\lim _{n \rightarrow \infty} X_{n}(0)=x_{0}$, and $X(t)$ satisfies

$$
X(t)=X(0)+\int_{0}^{t} F(X(s)) d s
$$


for all $T \geq 0$. Then

$$
\lim _{n \rightarrow \infty} \sup _{s \in[0, T]}\left|X_{n}(s)-X(s)\right|=0 \quad \text { a.s. }
$$

5.2. Hydordynamic limit for cross-multiplicative coalescent processes. Fix integers $K_{1}>0$ and $K_{2}>0$, and let $R:=R\left(K_{1}, K_{2}\right)=\left\{1,2, \ldots, K_{1}\right\} \times\left\{1,2, \ldots, K_{2}\right\}$. Let $e_{\mathbf{i}}$ be the standard basis vectors in $\mathbb{R}^{K_{1} K_{2}}$, enumerated by $\mathbf{i}=\left[\begin{array}{l}i_{1} \\ i_{2}\end{array}\right] \in R$. Consider a restriction to $\left[\begin{array}{l}i_{1} \\ i_{2}\end{array}\right] \in R$ of a Marcus-Lushnikov process $\zeta_{i_{1}, i_{2}}(t)$ with the cross-multiplicative kernel. Let

$$
\hat{X}_{n}(t)=\left\{\zeta_{i_{1}, i_{2}}^{[n]}(t)\right\}_{\mathbf{i} \in R}
$$

with the initial conditions $\hat{X}_{n}(0)=\alpha[n] e_{0^{\prime}}+\beta[n] e_{0^{\prime \prime}}$, where $0^{\prime}=\left[\begin{array}{l}1 \\ 0\end{array}\right]$ and $0^{\prime \prime}=\left[\begin{array}{l}0 \\ 1\end{array}\right]$.

We observe the following transition rates of $\hat{X}_{n}(t)$ stated as in (5.1). Let $x=$ $\sum_{\mathbf{i} \in R} x_{\mathbf{i}} e_{\mathbf{i}}$. Then, for any $\mathbf{i}$ and $\mathbf{j}$ in $R$, the change vector $\ell=-e_{\mathbf{i}}-e_{\mathbf{j}}+\mathbf{1}_{\{\mathbf{i}+\mathbf{j} \in R\}} e_{\mathbf{i}+\mathbf{j}}$ corresponding to a merger of clusters of respective weights $\mathbf{i}$ and $\mathbf{j}$ is assigned the rate

$$
q^{(n)}(x, x+\ell)=\frac{1}{n}\left(i_{1} j_{2}+i_{2} j_{1}\right) x_{\mathbf{i}} x_{\mathbf{j}}=n \beta_{\ell}(x),
$$

where $\beta_{\ell}(x)=\left(i_{1} j_{2}+i_{2} j_{1}\right) x_{\mathbf{i}} x_{\mathbf{j}}$.

For a given $\mathbf{i} \in R$, the change vector $\ell=-e_{\mathbf{i}}$ corresponding to the merger of clusters whose weight vector is $\mathbf{i}$ with clusters whose weight vectors are not in $R$ is assigned the rate

$$
\begin{aligned}
q^{(n)}(x, x+\ell) & =\frac{1}{n}\left[i_{1} x_{\mathbf{i}}\left(\beta[n]-\sum_{\mathbf{j} \in R} j_{2} x_{\mathbf{j}}\right)+i_{2} x_{\mathbf{i}}\left(\alpha[n]-\sum_{\mathbf{j} \in R} j_{1} x_{\mathbf{j}}\right)\right] \\
& =n\left[\beta_{\ell}(x)+O\left(\frac{1}{n}\right)\right],
\end{aligned}
$$

where $\beta_{\ell}(x)=i_{1} x_{\mathbf{i}}\left(\beta-\sum_{\mathbf{j} \in R} j_{2} x_{\mathbf{j}}\right)+i_{2} x_{\mathbf{i}}\left(\alpha-\sum_{\mathbf{j} \in R} j_{1} x_{\mathbf{j}}\right)$.

Thus, by Theorem 5.1, $X_{n}(t)$ converges to $X(t)$ as in (5.4), where $X(t)$ satisfies (5.3) with

$$
\begin{aligned}
F(x):=\sum_{\ell} \ell \beta_{\ell}(x)= & \frac{1}{2} \sum_{\mathbf{i}, \mathbf{j} \in R}\left[-e_{\mathbf{i}}-e_{\mathbf{j}}+\mathbf{1}_{\{\mathbf{i}+\mathbf{j} \in R\}} e_{\mathbf{i}+\mathbf{j}}\right]\left(i_{1} j_{2}+i_{2} j_{1}\right) x_{\mathbf{i}} x_{\mathbf{j}} \\
& -\sum_{\mathbf{i} \in R} e_{\mathbf{i}} i_{1} x_{\mathbf{i}}\left(\beta-\sum_{\mathbf{j} \in R} j_{2} x_{\mathbf{j}}\right)-\sum_{\mathbf{i} \in R} e_{\mathbf{i}} i_{2} x_{\mathbf{i}}\left(\alpha-\sum_{\mathbf{j} \in R} j_{1} x_{\mathbf{j}}\right) \\
= & \sum_{\mathbf{i} \in R} e_{\mathbf{i}}\left(-\left(\beta i_{1}+\alpha i_{2}\right) x_{\mathbf{i}}+\frac{1}{2} \sum_{\ell, \mathbf{k}: \ell+\mathbf{k}=\mathbf{i}}\left(\ell_{1} k_{2}+\ell_{2} k_{1}\right) x_{\ell} x_{\mathbf{k}}\right)
\end{aligned}
$$

for a fixed $T>0$. The system of equations (5.3) with $F(x)$ given in (5.5) will yield the reduced system of Smoluckowski coagulation equations (3.5). So, for a fixed a 
pair of positive integers $K_{1}$ and $K_{2}$, and a fixed real number $T>0$,

$$
\lim _{n \rightarrow \infty} \sup _{s \in[0, T]}\left|n^{-1} \zeta_{i_{1}, i_{2}}^{[n]}(s)-\zeta_{i_{1}, i_{2}}(s)\right|=0 \quad \text { a.s. }
$$

for all $\left[\begin{array}{l}i_{1} \\ i_{2}\end{array}\right] \in R$. Consequently,

$$
\lim _{n \rightarrow \infty} \sup _{s \in[0, T]}\left|n^{-1} \sum_{\substack{1 \leq i_{1} \leq K_{1} \\ 1 \leq i_{2} \leq K_{2}}} \zeta_{i_{1}, i_{2}}^{[n]}(s)-\sum_{\substack{1 \leq i_{1} \leq K_{1} \\ 1 \leq i_{2} \leq K_{2}}} \zeta_{i_{1}, i_{2}}(s)\right|=0 \quad \text { a.s. }
$$

5.3. Central Limit Theorem and related results. The usefulness of the framework set in Kurtz (1981) and Ethier and Kurtz (1986) for proving weak convergence is that the law of large numbers Theorem 5.1 is enhanced with the corresponding central limit theorem (see Theorem 5.2 below) and the large deviation theory in Feng and Kurtz (2006). The following central limit theorem is established in Theorem 8.2 of Kurtz (1981) and Theorem 2.3 in Chapter 11 of Ethier and Kurtz (1986).

Theorem 5.2. Suppose for all compact $\mathcal{K} \subset \mathbb{R}^{d}$,

$$
\sum_{\ell}|\ell|^{2} \sup _{x \in \mathcal{K}} \beta_{\ell}(x)<\infty
$$

and that the $\beta_{\ell}$ and $\partial F$ are continuous. Suppose $X_{n}$ and $X$ are as in Theorem 5.1, and suppose $V_{n}=\sqrt{n}\left(X_{n}-X\right)$ is such that $\lim _{n \rightarrow \infty} V_{n}(0)=V(0)$, where $V(0)$ is a constant. Then $V_{n}$ converges in distribution to $V$, which is the solution of

$$
V(t)=V(0)+U(t)+\int_{0}^{t} \partial F(X(s)) V(s) d s,
$$

where $U(t)$ is a Gaussian process and $\partial F(X(s))=\left(\partial_{j} F_{i}(X(s))\right)_{i, j}$.

The proof of Theorem 5.2 is based on representing $V_{n}(t)$ as follows. Let $Y_{\ell}$ be independent Poisson processes with rate one. Then,

$$
V_{n}(t)=V_{n}(0)+U_{n}(t)+\int_{0}^{t} \sqrt{n}\left(F\left(X_{n}(s)\right)-F(X(s))\right) d s,
$$

where

$$
U_{n}(t)=\sum_{\ell} \ell W_{\ell}^{(n)}\left(\int_{0}^{t} \beta_{\ell}\left(X_{n}(s)\right) d s\right),
$$

$W_{\ell}^{(n)}(u)=n^{-1 / 2} \hat{Y}_{\ell}(n u)$, and $\hat{Y}_{\ell}(u):=Y_{\ell}(u)-u$ are centralized Poisson processes.

Next, we will use formula (5.10) in order to derive an upper bound (5.11) on probability $P\left(\left|X_{n}(T)-X(T)\right| \geq \delta\right)$. Let us consider a simple case of a density dependent population process on $\mathbb{R}^{d}$ for which the following three conditions are satisfied.

i: $V_{n}=\sqrt{n}\left(X_{n}-X\right)$ is such that $\lim _{n \rightarrow \infty} V_{n}(0)=V(0)$.

ii: Both $X_{n}(t)$ and $X(t)$ live on a compact set $\mathcal{K}$.

iii: There are finitely many vectors $\ell \in \mathbb{R}^{d}$ such that $\beta_{\ell}(x)>0$ for some $x \in \mathcal{K}$. 
Notice that the above conditions are satisfied for the Marcus-Lushnikov processes with the cross-multiplicative kernel as in Subsection 5.2. Specifically, for a given $m>0$, let

$$
\mathcal{K}_{m}=\left\{x \in \mathbb{R}_{+}^{d}: \sum_{i} x_{i} \leq m\right\} .
$$

Then, in Subsection 5.2, we have $X_{n}(t), X(t) \in \mathcal{K}_{m}$ for $m>\alpha+\beta$.

Proposition 5.3. Assuming the above conditions i-iii are satisfied together with the Lipschitz continuity conditions (5.2), we have

$$
P\left(\left|X_{n}(T)-X(T)\right| \geq \delta\right)=O\left(n^{-2}\right) .
$$

Proof: Here,

$$
\sqrt{n}\left|F\left(X_{n}(s)\right)-F(X(s))\right| \leq \sqrt{n} M_{\mathcal{K}}\left|X_{n}(s)-X(s)\right|=M_{\mathcal{K}}\left|V_{n}(s)\right|
$$

and for a fixed $T>0$ and any $t \leq T$,

$$
\left|V_{n}(0)+U_{n}(t)\right| \leq \varepsilon_{n}(T):=\left|V_{n}(0)\right|+\sum_{\ell}|\ell| \max \left\{\left|W_{\ell}^{(n)}(s)\right|: s \in\left[0, T \sup _{x \in \mathcal{K}} \beta_{\ell}(x)\right]\right\} .
$$

Hence, for a fixed $T>0$, equation (5.10) implies the following inequality,

$$
\left|V_{n}(t)\right| \leq \varepsilon_{n}(T)+M_{\mathcal{K}} \int_{0}^{t}\left|V_{n}(s)\right| d s \quad \text { for all } t \in[0, T] .
$$

Then, by Grönwall's inequality (see Appendix 5 in Ethier and Kurtz, 1986),

$$
\left|V_{n}(t)\right| \leq \varepsilon_{n}(T) e^{M_{\mathcal{K}} t}
$$

In particular, we use equation (5.12) together with Markov inequality to obtain the following simple bound for any $\delta>0$,

$$
P\left(\left|X_{n}(T)-X(T)\right| \geq \delta\right) \leq \frac{V_{n}^{4}(T)}{n^{2} \delta^{4}} \leq \frac{E\left[\varepsilon_{n}^{4}(T)\right] e^{4 M_{\mathcal{K}} T}}{n^{2} \delta^{4}} .
$$

Here, for any fixed real $S>0$, integer $r>0$, and any real $\lambda>0$, we have by Doob's martingale inequality,

$P\left(\max _{s \in[0, S]}\left|W_{\ell}^{(n)}(s)\right|^{r} \geq \lambda\right)=P\left(\max _{s \in[0, S]}\left|W_{\ell}^{(n)}(s)\right| \geq \lambda^{1 / r}\right) \leq \frac{E\left[\left(W_{\ell}^{(n)}(S)\right)^{2+2 r}\right]}{\lambda^{2+2 / r}}$

as $\left|W_{\ell}^{(n)}(s)\right|$ is a non-negative sub-martingale. Therefore,

$$
\begin{aligned}
E\left[\max _{s \in[0, S]}\left|W_{\ell}^{(n)}(s)\right|^{r}\right] & \leq 1+\int_{1}^{\infty} P\left(\max _{s \in[0, S]}\left|W_{\ell}^{(n)}(s)\right|^{r} \geq \lambda\right) d \lambda \\
& \leq 1+(1+2 / r) E\left[\left(W_{\ell}^{(n)}(S)\right)^{2+2 r}\right],
\end{aligned}
$$

where by the classical central limit theorem,

$$
\lim _{n \rightarrow \infty} E\left[\left(W_{\ell}^{(n)}(S)\right)^{2+2 r}\right]=S^{1+r} E\left[Z^{2+2 r}\right], \quad Z \text { - standard normal r.v. }
$$

Thus, $E\left[\varepsilon_{n}^{4}(T)\right]=O(1)$, and (5.11) follows from (5.13). 


\section{Discussion: generalizations and open problems.}

In this paper we considered an important example of coagulation ODEs obtained as a hydrodynamic limit of a Marcus-Lushnikov process that tracks the merger history of a coalescent process with two dimensional weight vectors. The coagulation equations and gelation in the Marcus-Lushnikov dynamics for other coalescent processes with multidimensional weight vectors is on its own an interesting object of studies. As a natural next step, one may consider a generalization of the existing results Aldous (1998); Jeon (1998, 1999); Norris (1999); Escobedo et al. (2002); Fournier and Giet (2004); Fournier and Laurençot (2009) on gelation phenomenon for vector weighted processes.

An extension of the application to minimal spanning trees may come from an observation that the convergence rates in the hydrodynamic limit yield the central limit theorem for $L_{n}$ on $K_{\alpha[n], \beta[n]}$ similar to the central limit theorem for $L_{n}$ on $K_{n}$ proved in Janson (1995). Specifically, we hope to apply Theorem 5.2 in the analysis. Moreover, similarly to Cooper et al. (2016), it is possible to examine the second and third order terms in $L_{n}$.

\section{References}

Aldous, D. Emergence of the giant component in special Marcus-Lushnikov processes. Random Structures Algorithms, 12 (2), 179-196 (1998).

Aldous, D. J. Deterministic and stochastic models for coalescence (aggregation and coagulation): a review of the mean-field theory for probabilists. Bernoulli, 5 (1), 3-48 (1999). MR1673235.

Austin, T. L. The enumeration of point labelled chromatic graphs and trees. Canadian J. Math., 12, 535-545 (1960). MR139544.

Berestycki, N. Recent progress in coalescent theory, volume 16 of Ensaios Matemáticos [Mathematical Surveys]. Sociedade Brasileira de Matemática, Rio de Janeiro (2009). ISBN 978-85-85818-40-1. MR2574323.

Bertoin, J. Random fragmentation and coagulation processes, volume 102 of Cambridge Studies in Advanced Mathematics. Cambridge University Press, Cambridge (2006). ISBN 978-0-521-86728-3; 0-521-86728-2. MR2253162.

Beveridge, A., Frieze, A., and McDiarmid, C. Random minimum length spanning trees in regular graphs. Combinatorica, 18 (3), 311-333 (1998). MR1721947.

Comtet, L. Advanced combinatorics. The art of finite and infinite expansions. D. Reidel Publishing Co., Dordrecht, enlarged edition (1974). ISBN 90-277-0441-4. MR0460128.

Cooper, C., Frieze, A., Ince, N., Janson, S., and Spencer, J. On the length of a random minimum spanning tree. Combin. Probab. Comput., 25 (1), 89-107 (2016). MR3438287.

Erdős, P. and Rényi, A. On the evolution of random graphs. Publ. Math. Inst. Hung. Acad. Sci, 5 (1), 17-60 (1960). MR0125031.

Ernst, M. H., Hendriks, E. M., and Ziff, R. M. Critical kinetics near the gelation transition. J. Phys. A, 15 (12), L743-L747 (1982). MR682327.

Escobedo, M., Mischler, S., and Perthame, B. Gelation in coagulation and fragmentation models. Comm. Math. Phys., 231 (1), 157-188 (2002). MR1947695.

Ethier, S. N. and Kurtz, T. G. Markov processes. Characterization and convergence. Wiley Series in Probability and Mathematical Statistics: Probability and 
Mathematical Statistics. John Wiley \& Sons, Inc., New York (1986). ISBN 0471-08186-8. MR838085.

Evans, S. N. and Pitman, J. Construction of Markovian coalescents. Ann. Inst. H. Poincaré Probab. Statist., 34 (3), 339-383 (1998). MR1625867.

Feng, J. and Kurtz, T. G. Large deviations for stochastic processes, volume 131 of Mathematical Surveys and Monographs. American Mathematical Society, Providence, RI (2006). ISBN 978-0-8218-4145-7; 0-8218-4145-9. MR2260560.

Flory, P. J. Principles of polymer chemistry. Cornell University Press (1953).

Fournier, N. and Giet, J.-S. Convergence of the Marcus-Lushnikov process. Methodol. Comput. Appl. Probab., 6 (2), 219-231 (2004). MR2035293.

Fournier, N. and Laurençot, P. Marcus-Lushnikov processes, Smoluchowski's and Flory's models. Stochastic Process. Appl., 119 (1), 167-189 (2009). MR2485023.

Frieze, A. M. On the value of a random minimum spanning tree problem. Discrete Appl. Math., 10 (1), 47-56 (1985). MR770868.

Frieze, A. M. and McDiarmid, C. J. H. On random minimum length spanning trees. Combinatorica, 9 (4), 363-374 (1989). MR1054012.

Huang, F. and Liu, B. The Abel-type polynomial identities. Electron. J. Combin., 17 (1), Research Paper 10, 7 (2010). MR2578905.

Janson, S. The minimal spanning tree in a complete graph and a functional limit theorem for trees in a random graph. Random Structures Algorithms, 7 (4), 337-355 (1995). MR1369071.

Jeon, I. Existence of gelling solutions for coagulation-fragmentation equations. Comm. Math. Phys., 194 (3), 541-567 (1998). MR1631473.

Jeon, I. Spouge's conjecture on complete and instantaneous gelation. J. Statist. Phys., 96 (5-6), 1049-1070 (1999). MR1722986.

Krapivsky, P. L. and Ben-Naim, E. Aggregation with multiple conservation laws. Phys. Rev. E, 53, 291-298 (1996). DOI: 10.1103/PhysRevE.53.291.

Kurtz, T. G. Approximation of population processes, volume 36 of CBMS-NSF Regional Conference Series in Applied Mathematics. Society for Industrial and Applied Mathematics (SIAM), Philadelphia, Pa. (1981). ISBN 0-89871-169-X. MR610982.

Lushnikov, A. A. Coagulation in finite systems. Journal of Colloid and Interface Science, 65 (2), 276-285 (1978). DOI: 10.1016/0021-9797(78)90158-3.

Marcus, A. H. Stochastic coalescence. Technometrics, 10, 133-143 (1968). MR223151.

McLeod, J. B. On an infinite set of non-linear differential equations. Quart. J. Math. Oxford Ser. (2), 13, 119-128 (1962). MR139822.

Norris, J. R. Smoluchowski's coagulation equation: uniqueness, nonuniqueness and a hydrodynamic limit for the stochastic coalescent. Ann. Appl. Probab., 9 (1), 78-109 (1999). MR1682596.

Pitman, J. Combinatorial stochastic processes, volume 1875 of Lecture Notes in Mathematics. Springer-Verlag, Berlin (2006). ISBN 978-3-540-30990-1; 3-54030990-X. MR2245368.

Rezakhanlou, F. Gelation for Marcus-Lushnikov process. Ann. Probab., 41 (3B), 1806-1830 (2013). MR3098059.

Riordan, J. Combinatorial identities. John Wiley \& Sons, Inc., New York-LondonSydney (1968). MR0231725. 
Smoluchowski, M. Brownsche Molekularbewegung und Koagulation von Kolloidteilchen. Physik. Zeit., 17, 585-599 (1916).

Spouge, J. L. Solutions and critical times for the monodisperse coagulation equation when $a_{i j}=A+B(i+j)+C i j$. J. Phys. A, 16 (4), 767-773 (1983). MR706194.

van Dongen, P. G. J. and Ernst, M. H. On the occurrence of a gelation transition in Smoluchowski's coagulation equation. J. Statist. Phys., 44 (5-6), 785-792 (1986). MR858257.

Vigil, R. D. and Ziff, R. M. On the scaling theory of two-component aggregation. Chemical Engineering Science, 53 (9), 1725-1729 (1998). DOI: 10.1016/S00092509(98)00016-5.

Ziff, R. M. Kinetics of polymerization. J. Statist. Phys., 23 (2), 241-263 (1980). MR586509. 